\title{
VISCOSITY SOLUTIONS FOR AN OPTIMAL CONTROL PROBLEM WITH PREISACH HYSTERESIS NONLINEARITIES
}

\author{
FABiO BAGAGIOLO ${ }^{1}$
}

\begin{abstract}
We study a finite horizon problem for a system whose evolution is governed by a controlled ordinary differential equation, which takes also account of a hysteretic component: namely, the output of a Preisach operator of hysteresis. We derive a discontinuous infinite dimensional Hamilton-Jacobi equation and prove that, under fairly general hypotheses, the value function is the unique bounded and uniformly continuous viscosity solution of the corresponding Cauchy problem.
\end{abstract}

Mathematics Subject Classification. 47J40, 49J15, 49L20, 49L25.

Received September 4, 2002. Revised November 4, 2003.

\section{INTRODUCTION}

The Preisach operator is a very important and well-studied mathematical model for describing hysteresis phenomena (i.e. rate independent memory effects). It is probably the most used model for representing hysteresis from physical and natural sciences, as for instance ferromagnetic hysteresis, hysteresis in filtration through porous media, phase transitions, shape memory alloys and smart structure materials. It has also applications to biology and economics. Its importance is also due to the fact it has two characteristic properties, the so-called deletion and congruency properties. This means that, whenever a general input-output hysteresis relationship satisfies those two properties (which are experimentally observable), then it is representable by a Preisach operator.

Many mathematical works have been done on the analytical properties of the Preisach model, and on its connection with other fields, in particular with the theory of evolutionary PDEs (see the monographs KrasnoselskiiPokrovskii [12], Mayergoyz [18], Visintin [21], Brokate-Sprekels [9], Krejci [13], and Della Torre [11], for the analytical study as well as for applications and extensions). Also, several control problems, for systems with hysteresis of Preisach type, have been studied.

The present paper, at least for our knowledge, is the first one concerning with an optimal control problem for a system with Preisach hysteresis, within the theory of viscosity solutions for Hamilton-Jacobi equations. In particular, we derive and study the infinite dimensional and discontinuous Hamilton-Jacobi-Bellman equation, for a finite horizon optimal control problem, given by the following controlled ODE system

$$
\begin{cases}y^{\prime}(t)=f(y(t), w(t), \alpha(t)) & t>0 \\ w(t)=\mathcal{H}_{\mu}\left[y, \xi_{0}\right](t) & t \geq 0 \\ y(0)=y_{0}, & \end{cases}
$$

Keywords and phrases. Hysteresis, optimal control, dynamic programming, viscosity solutions.

1 Dipartimento di Matematica, Università di Trento, Via Sommarive 14, 38050 Povo-Trento, Italy;

e-mail: bagagiol@science.unitn.it 
and by the following cost functional and value function

$$
\begin{aligned}
J\left(y_{0}, \xi_{0}, t, \alpha\right) & :=\int_{0}^{t} \mathrm{e}^{-\lambda s} \ell(y(s), w(s), \alpha(s)) \mathrm{d} s+\mathrm{e}^{-\lambda t} h(y(t), w(t)) ; \\
V\left(y_{0}, \xi_{0}, t\right) & :=\inf _{\alpha \in \mathcal{A}} J\left(y_{0}, \xi_{0}, t, \alpha\right) .
\end{aligned}
$$

Here, $\mathcal{A}$ is the set of measurable controls $\alpha:[0,+\infty[\rightarrow A$, with $A$ compact; the scalar functions $y(\cdot)$ and $w(\cdot)$ are, respectively, the input and the output of the Preisach hysteresis operator $\mathcal{H}_{\mu}$, with initial configuration $\xi_{0}$. Our aim is to characterize the value function as the unique viscosity solution, in an adapted sense, of a suitable Hamilton-Jacobi equation.

Control and optimal control problems for systems with hysteresis are of course of great importance for applications (see for instance the book by Tao and Kokotovic [20], where some examples are illustrated). Sometimes, it is important to optimally control a system where hysteresis cannot be neglected; other times it is the hysteresis phenomenon itself what should be optimally controlled, in order to prevent undesirable effects.

An optimal control problem for ODE with the Preisach hysteresis model is studied by Brokate in [8], concerning necessary conditions for optimality. In that work the author does not apply the dynamic programming method. Belbas and Mayergoyz studied in [6] an optimal control problem for ODE with hysteresis of Madelung type. They derive the dynamic programming principle, but they do not derive the corresponding HamiltonJacobi equation. Recently, in [7], they write a set-valued Hamilton-Jacobi equation for an optimal control problem with Preisach hysteresis. However, they do not perform a viscosity solutions analysis of that equation. In [15], Lenhart, Seidman and Yong studied an optimal control problem for an ODE system with switching hysteresis along one component of the three dimensional vectorial input. They are mainly concerned with the existence of optimal controls. In [19], Tan and Baras apply the viscosity solutions technique to the continuous and finite dimensional Hamilton-Jacobi equation for an optimal control problem with Duhem type hysteresis.

The works $[6,7,19]$ explicitly refer to applications to magnetostrictive actuators, whereas the work [15] is concerned with applications to a problem of bioremediation.

The Preisach operator consists of a superposition of (possibly infinite) rectangular hysteresis loops, the socalled delayed relays. Every delayed relay gives a switching rule between a continuous time-dependent scalar input and a discontinuous time-dependent scalar output which may assume only the values 1 or -1 . Every delayed relay is characterized by the thresholds $\rho_{1}<\rho_{2}$, respectively for switching down (from 1 to -1 ) and for switching up (from -1 to 1 ) (see Fig. 1), and hence it uniquely corresponds to a point of the so-called Preisach plane

$$
\mathcal{P}:=\left\{\rho=\left(\rho_{1}, \rho_{2}\right) \mid \rho_{1}<\rho_{2}\right\} .
$$

Given a signed finite Borel measure $\mu$ on $\mathcal{P}$, for every continuous input $u \in C^{0}([0,+\infty[)$, and for every initial configuration $\xi: \mathcal{P} \rightarrow\{1,-1\}$ (which represents the initial state of all relays), the output $w(\cdot)=\mathcal{H}_{\mu}[u, \xi](\cdot)$ of the Preisach hysteresis model is constructed as the average, with respect to $\mu$, of the outputs of all delayed relays with the same input $u$.

The model exhibits the evolution of the so-called internal variable, which, for every $t$ is a function on the Preisach plane, which may assume only the values 1 and -1

$$
t \mapsto \xi_{t}: \mathcal{P} \rightarrow\{1,-1\} .
$$

Under general hypotheses on $\mu$, the output $w$ is continuous. Moreover, for a suitable measure $\nu$ on $\mathcal{P}$, the map $(u, \xi) \mapsto \mathcal{H}_{\mu}[u, \xi](\cdot)$ is Lipschitz continuous for the topologies of $C^{0}([0, T]) \times L^{2}(\mathcal{P}, \nu)$ and $C^{0}([0, T])$.

Let us come back to the optimal control problem. The state of the problem is the couple $(y, \xi)$, where $y$ is a real number and $\xi$ is a function on the Preisach plane $\mathcal{P}$. Note that we are working with two measures on $\mathcal{P}: \mu$, which is signed and enters in the definition of the Preisach operator: it is a datum of the problem; $\nu$, which is positive and gives the space $L^{2}(\mathcal{P}, \nu)$ into which we are going to embed our problem: its choice is at our disposal. Of course, $\mu$ and $\nu$ should be somehow related. 
By the regularity of $\mathcal{H}_{\mu},(1.1)$ has a unique solution, and, since $\mathcal{H}_{\mu}$ satisfies a semigroup property, we easily derive the dynamic programming principle for the value function $V$. From this, we deduce a Hamilton-Jacobi equation satisfied by $V$ in the (adapted) viscosity sense. Such equation consists of a usual "finite dimensional part" (which involves the derivatives with respect to $t$ and to $y$ ), and of a "infinite dimensional" part (which involves the Fréchet differentials with respect to $\left.\xi \in L^{2}(\mathcal{P}, \nu)\right)$.

After a suitable correspondence with the set of maximal antimonotone graphs on $\mathcal{P}$, and after a suitable change of variables, the probably "natural" setting for the functions $\xi$ is a subset of a space of continuous functions. But the space of continuous functions, with the $L^{\infty}$-norm, is not a suitable space for viscosity solutions technique. This is in particular due to the fact that it does not have suitably regular "penalization functions", which are used for the comparison results. Another possible choice of a space into which embed our problem is $L^{1}(\mathcal{P}, \nu)$. But, as before, it is not a good space for the viscosity solutions technique. Hence, we embed our problem into $L^{2}(\mathcal{P}, \nu)$ (where the square of the norm is a differentiable penalization function).

However, the choice of $L^{2}(\mathcal{P}, \nu)$ brings some other problems. One problem, which is also due to the Preisach hysteresis model, is the fact that the evolution $t \mapsto \xi_{t}$, given by (1.1) and (1.2), does not belong to $W^{1,1}\left((0, T), L^{2}(\mathcal{P}, \nu)\right)$. Indeed, using again the correspondence between the internal variables $\xi$ and the antimonotone graphs on $\mathcal{P}$, if the input is increasing (respectively, decreasing), then the right derivative of $t \mapsto \xi_{t}$ is related to the possible final horizontal segment (respectively, final vertical segment) which links the corresponding antimonotone graph to the line $\rho_{1}=\rho_{2}$ (see Fig. 2). Hence, in the Hamilton-Jacobi equation, we are lead to consider a quantity $T^{u}$ (respectively, $T^{l}$ ), which in some sense is the trace from above (respectively, from the left) on such a final horizontal segment (respectively, final vertical segment) of a suitable combination of the first and second Fréchet differentials of the test function. The presence of the second Fréchet differential is due to the fact that we have to control the difference of the values that a test function takes on two elements of $L^{2}(\mathcal{P}, \nu)$, which differ only for the behavior on a thin rectangle. To this end, we need to work with the second order Taylor formula.

A second problem is the fact that the two quantities $T^{u}=T^{u}[\phi](y, \xi)$ and $T^{l}=T^{l}[\phi](y, \xi)$, which depend on the test function $\phi$ and on the state-space point $(y, \xi)$, are not continuous for the topology of $(y, \xi) \in$ $\mathbb{R} \times L^{2}(\mathcal{P}, \nu)$. This fact leads us to consider a "lower" and an "upper" Hamilton-Jacobi equation, given by the two Hamiltonians

$$
\begin{aligned}
H^{-}\left(y, \xi, \phi_{y}, D \phi, D^{2} \phi\right):= & \sup _{a \in A}\left\{-\phi_{y} f(y, w, a)-\left(f^{+}(y, w, a)\left(T^{u}[\phi](y, \xi)\right)^{+}\right.\right. \\
& \left.\left.+f^{-}(y, w, a)\left(T^{l}[\phi](y, \xi)\right)^{-}\right)-\ell(y, w, a)\right\} \\
H^{+}\left(y, \xi, \phi_{y}, D \phi, D^{2} \phi\right):= & \sup _{a \in A}\left\{-\phi_{y} f(y, w, a)+\left(f^{+}(y, w, a)\left(T^{u}[\phi](y, \xi)\right)^{-}\right.\right. \\
& \left.\left.+f^{-}(y, w, a)\left(T^{l}[\phi](y, \xi)\right)^{+}\right)-\ell(y, w, a)\right\}
\end{aligned}
$$

where $(\cdot)^{+}$and $(\cdot)^{-}$are the positive and the negative part, and $w=\int_{\mathcal{P}} \xi \mathrm{d} \mu$.

This is of course coherent with the theory of viscosity solutions for discontinuous Hamilton-Jacobi equations, where, typically, the definition of viscosity solution involves the upper and lower semicontinuous envelopes of the Hamiltonian. The discontinuous part of the Hamiltonian is often somehow related to the boundary conditions in the viscosity sense. Also in our case, the discontinuous "infinite dimensional" components of the Hamiltonians, in some sense, play the role of a boundary condition, where the boundary is given by the couples $(y, \xi)$ corresponding to maximal antimonotone graphs with final horizontal or final vertical segment (if such segments do not appear, then $T^{u}$ and $T^{l}$ vanish). The problem here is that such "boundary" is dense for the topology of $\mathbb{R} \times L^{2}(\mathcal{P}, \nu)$ in the state-space, and moreover the topology of $L^{2}(\mathcal{P}, \nu)$ is not strong enough in order to force the couple $(y, \xi)$ to not belong to such "boundary". However, under fairly general hypotheses on the measure $\nu$ (without any pretension of sharpness), and working with suitable test functions in the usual comparison technique, we are able to force the signs of $T^{u}$ and $T^{l}$, in order to make them vanishing inside the Hamiltonians. This permits us to work only with the continuous finite dimensional part. In this effort, we are 
also helped by the fact that the state-space has the property that every bounded subset has compact closure in $\mathbb{R} \times L^{2}(\mathcal{P}, \nu)$, and hence we do not need to use any variational principle.

Our main result gives, under general hypotheses, the uniqueness of the value function $V$ as viscosity solution of the Cauchy problem

$$
\left\{\begin{array}{l}
V_{t}(y, \xi, t)+\lambda V(y, \xi, t)+H^{-}\left(y, \xi, V_{y}(y, \xi, t), D V(y, \xi, t), D^{2} V(y, \xi, t)\right) \leq 0 \\
V_{t}(y, \xi, t)+\lambda V(y, \xi, t)+H^{+}\left(y, \xi, V_{y}(y \xi, t), D V(y, \xi, t), D^{2} V(y, \xi, t)\right) \geq 0 \\
V(y, \xi, 0)=h(y, w)
\end{array}\right.
$$

with test functions from a suitable subset of $C^{1}(\mathbb{R} \times] 0,+\infty[) \times C^{2}\left(L^{2}(\mathcal{P}, \nu)\right)$.

The model described up to now is a "scalar" model, in the sense that the input and the output of the Preisach operator are scalar functions; moreover, the scalar input is the directly controlled quantity (i.e. $y$ in (1.1)). The main reason for that is the fact that the Preisach model is a scalar hysteresis operator (i.e. scalar input and scalar output). Nevertheless, it is probably the most important, interesting and versatile hysteresis model. Some extensions of the Preisach model to vectorial inputs and vectorial outputs are indeed studied, especially in connection with ferromagnetic hysteresis. But the results are less satisfactory than the scalar case, both from the analytical and from the applications point of view. However, most of the possible extensions are constructed starting from the scalar Preisach model. For instance, an extension may be given by an average of the outputs of scalar Preisach operators, each one of them respectively acting on a component of the vectorial input. Hence, the analysis we perform in this paper for the "scalar" optimal control problem with Preisach hysteresis, is certainly useful for studying many possible extensions to the vectorial case. Indeed, what is important and novel in our analysis, is the study of the evolution of the infinite dimensional internal variable of the Preisach model, in connection with the dynamic programming principle and the Hamilton-Jacobi theory. To show this fact, we actually extend our results to a rather general vectorial control problem with Preisach-type hysteresis. In particular, this general case covers the situations studied by other authors.

We recall that the viscosity solutions theory for Hamilton-Jacobi equations, and its connection with optimal control problems, is a well known framework. We refer the reader to the book Bardi-Capuzzo Dolcetta [4] for the theory in finite dimension, to the works Crandall-Lions [10] and Lions [17] for results about the infinite dimensional case, and to Lions [16], Ishii [14] and Barles-Lions [5] for the case of discontinuous equations.

However, we point out that, from the viscosity solutions point of view, the infinite dimensional feature of our Hamilton-Jacobi equation is new. Indeed, it is given by the nonlinear input-output Preisach hysteresis relationship, and not by a PDE, as it is common in the known literature.

Finally we observe that the present author, in [1] and [2], studied two optimal control problems for ODE with hysteresis. In those papers, a Hamilton-Jacobi equation satisfied by the value function is derived and studied in the framework of viscosity solutions. However, those settings are different from the present one. In particular, in [2] the case of the Play/Prandtl-Ishlinskii model and in [1] the case of a finite sum of delayed relays are, respectively, studied (they respectively lead to a finite/infinite dimensional discontinuous Hamilton-Jacobi equation and to a suitably coupled system of finite dimensional continuous Hamilton-Jacobi equations). See also [3] for an extension of [1] to a vectorial case.

The plan of the paper is as follows. In Section 2, we describe the delayed relay and the Preisach model. In Section 3, we state the control problem. In Section 4, we rigorously derive the Hamilton-Jacobi equation satisfied by the value function. In Section 5 , we prove the uniqueness result. In Section 6 , we briefly study an extension to the vectorial case.

\section{The Preisach Model}

In this section we introduce the Preisach model of hysteresis. We essentially follow Visintin [21] (and we refer the reader to it for a more precise and rigorous description). However, we change somewhere definitions in order to fit better the control problem in the sequel. 


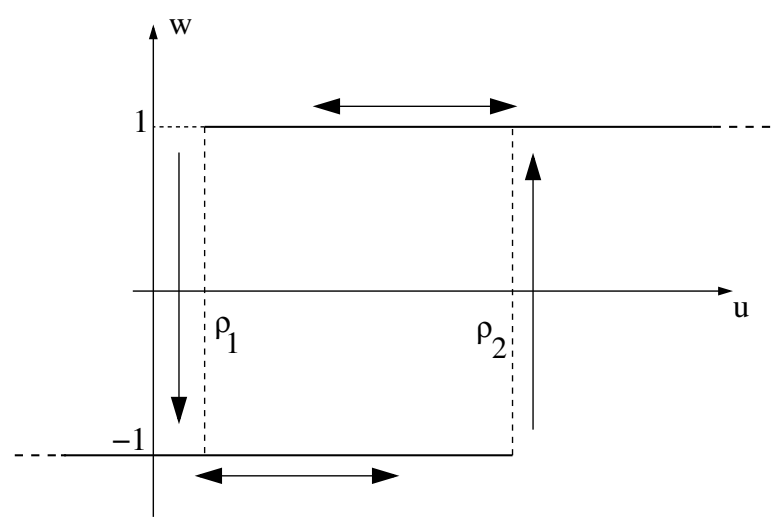

Figure 1. Delayed relay.

\subsection{The discontinuous delayed relay}

Let us fix two thresholds $\rho_{1}<\rho_{2}$ and consider a graph as in Figure 1.

It represents the relationship between a continuous scalar input $u(t)$ and a discontinuous output $w(t)$ which switches between the values 1 and -1 . The (delayed) switching rule is as follows. Let $u(0) \leq \rho_{1}$. Then $w(0)=-1$, and $w(t)=-1$ as long as $u(t)<\rho_{2}$; if at some instant $u$ reaches $\rho_{2}$, then $w$ switches up to 1 , where it remains as long as $u(t)>\rho_{1}$; if later, $u$ reaches $\rho_{1}$ then $w$ switches down to -1 ; and so on. Otherwise, if $u(0) \geq \rho_{2}$, then $w(0)=1$ and the evolution of $w$ is similarly described. Finally, if $\rho_{1}<u(0)<\rho_{2}$, then we have to give the initial value of $w$, because both 1 and -1 are admissible, and then the evolution follows the same rules as before. For any time interval $[0, T]$ and for every couple of thresholds $\rho=\left(\rho_{1}, \rho_{2}\right)$, we define the corresponding delayed relay operator in the following way

$$
h_{\rho}: C^{0}([0, T]) \times\{-1,1\} \rightarrow L^{\infty}(0, T), \quad(u, \xi) \mapsto h_{\rho}[u, \xi](\cdot):=w(\cdot),
$$

where $\xi$ (the initial value of the output) plays a role only if $\rho_{1}<u(0)<\rho_{2}$.

\subsection{The Preisach model}

The thresholds of the delayed relay operators form the so-called Preisach plane

$$
\mathcal{P}:=\left\{\rho=\left(\rho_{1}, \rho_{2}\right) \in \mathbb{R}^{2} \mid \rho_{1}<\rho_{2}\right\}
$$

We denote by $\mathcal{R}$ the family of Borel measurable functions $\mathcal{P} \rightarrow\{-1,1\}$, and by $\xi: \rho \mapsto \xi(\rho)$ a generic element of $\mathcal{R}$, which we intend to represent the initial configurations of all the delayed relays. We fix a finite signed Borel measure $\mu$ over $\mathcal{P}$, and introduce the corresponding Preisach operator

$$
\begin{aligned}
& \mathcal{H}_{\mu}: C^{0}([0, T]) \times \mathcal{R} \rightarrow L^{\infty}(0, T) \\
& w(t)=\mathcal{H}_{\mu}[u, \xi](t):=\int_{\mathcal{P}} h_{\rho}[u, \xi(\rho)](t) \mathrm{d} \mu(\rho) \quad \forall t \in[0, T]
\end{aligned}
$$

Setting $\xi_{0}(\rho)=\xi(\rho)$, the evolution $t \mapsto \xi_{t}$, with $\xi_{t}(\rho)=h_{\rho}\left[u, \xi_{0}\right](t)$, represents the evolution of the internal variables $\xi$. Note that, even if we know the evolution of the input $u$, the knowledge of the value of the output $w(t)$ is not enough for determining the evolution of $w$ in $] t, T]$; we need to know the value of the internal variable $\xi_{t}$, namely the configuration of all relays.

Looking at the Preisach plane, we can see an interesting and useful geometric interpretation of the evolution of the internal variables $\xi_{t}$. Denoting by $A^{+}(t)$ (respectively by $A^{-}(t)$ ) the subset of $\mathcal{P}$ given by the points 


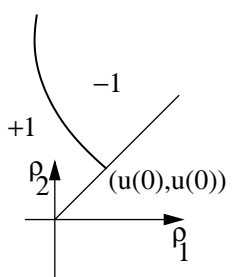

(i)

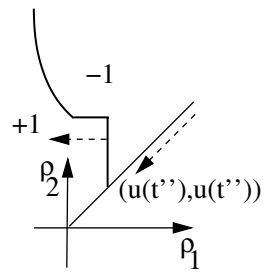

(iii)

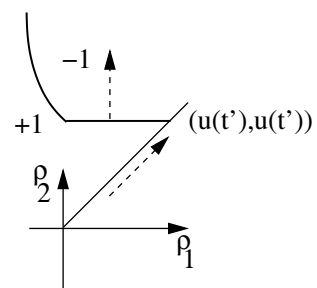

(ii)

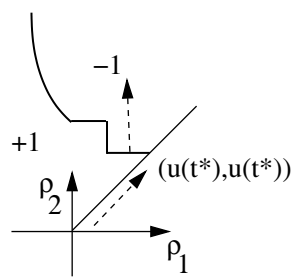

(iv)

Figure 2. Evolution on the Preisach plane.

$\rho$ corresponding to the relays which at the time $t$ are switched on 1, i.e. $\xi_{t}(\rho)=1$ (respectively -1 , i.e. $\left.\xi_{t}(\rho)=-1\right)$, then, by definition,

$$
\mathcal{H}_{\mu}[u, \xi](t)=\mu\left(A^{+}(t)\right)-\mu\left(A^{-}(t)\right)
$$

Let us fix an initial configuration $\xi$. Let us suppose that there exists a maximal antimonotone graph $B$ on $\overline{\mathcal{P}}$ such that all the relays corresponding to the points $\rho \in \mathcal{P}$ which are above (respectively below) $B$ are switched on -1 , i.e. $\xi(\rho)=-1$ (respectively on 1, i.e. $\xi(\rho)=1$ ), see Figure 2 i. The "end point" of the graph $B$ is the point $(u(0), u(0))$ on the line $\rho_{1}=\rho_{2}$, where $u(0)$ is the initial value of the input. Let us suppose that $u$ increases from $u(0)$ to $u\left(t^{\prime}\right)$. Then some relays switch from -1 to 1 (precisely, those relays whose upper threshold $\rho_{2}$ is reached by $u$ ), and the new configuration is represented by Figure 2ii: a horizontal segment is formed and is up-ward moving on the Preisach plane. Now, let us suppose that the input decreases from $u\left(t^{\prime}\right)$ to $u\left(t^{\prime \prime}\right)$. Then some relays switch from 1 to -1 (precisely, those relays whose lower threshold $\rho_{1}$ is reached by $u$ ), and the new configuration is represented by Figure 2iii: a vertical segment is formed and is left-ward moving on the Preisach plane. If later, the input increases again to a value $u\left(t^{*}\right)$, then the new configuration is represented by Figure 2iv.

A rigorous proof of the following result can be found in Visintin [21].

Theorem 2.1. Let us take an initial configuration $\xi$ which corresponds to a maximal antimonotone graph $B$ (as above explained). By applying an input $u \in C^{0}([0, T])$, at any time $t \in[0, T]$ we have a configuration on the Preisach plane given by a maximal antimonotone graph, which equals $B$ out of a compact set, and, near the line $\rho_{1}=\rho_{2}$, consists of at most a countable family of horizontal and vertical segments.

Remark 2.2. Note that we confine ourselves to the case of initial configurations corresponding to a maximal antimonotone graph. The reasons are the following. First of all note that, as in Theorem 2.1, we can say that, whichever the initial configuration is, possibly after a cycling evolution of the input, a "stair-shaped" maximal monotone graph in the Preisach plane is formed around the point $(u(t), u(t))$. Also, for the same reason, Theorem 2.1 assures that the set of maximal antimonotone graphs on $\mathcal{P}$ is invariant for the evolution of the internal variables. Moreover, if we suppose starting from a (ideal) situation which has never experienced past evolution (what may be called the "virgin state"), then a natural hypothesis is that the configuration corresponds to the symmetric maximal antimonotone graph $\left\{\left(\rho_{1}, \rho_{2}\right) \in \mathcal{P} \mid \rho_{1}=-\rho_{2}\right\}$. Finally, without starting from a configuration corresponding to a maximal antimonotone graph, some of the regularity properties of the Preisach model do not hold anymore. 
Definition 2.3. We denote by $\mathcal{B}$ the set of all maximal antimonotone graphs on $\overline{\mathcal{P}}$, and by $\mathcal{S}$ the set of all functions $\xi: \mathcal{P} \rightarrow\{-1,1\}$ such that there exists $B \in \mathcal{B}$ with $\xi \equiv 1$ (respectively $\xi \equiv-1$ ) below (respectively above) $B$. Hence there exists a bijection between $\mathcal{S}$ and $\mathcal{B}$. For every $\xi \in \mathcal{S}$ we denote by $B_{\xi} \in \mathcal{B}$ the corresponding maximal antimonotone graph. Moreover, we denote by $\mathcal{O}$ the set given by the couples $(y, \xi) \in$ $\mathbb{R} \times \mathcal{S}$ such that $(y, y) \in B_{\xi}$. Moreover, we say that a couple $(u, \xi) \in C^{0}([0, T]) \times \mathcal{S}$ is admissible if $(u(0), \xi) \in \mathcal{O}$.

Note that we do not care about the value of $\xi$ on the graph $B_{\xi}$ itself, indeed we will always use measures on $\mathcal{P}$ for which such graphs have measure zero.

Sometimes, it is useful to consider a new system of coordinates on $\mathcal{P}$ :

$$
\sigma_{1}:=\frac{\rho_{2}-\rho_{1}}{\sqrt{2}}, \quad \sigma_{2}:=\frac{\rho_{1}+\rho_{2}}{\sqrt{2}} .
$$

With respect to those coordinates, every element of $\mathcal{B}$ is the graph of a Lipschitz continuous function $\sigma_{1} \mapsto \sigma_{2}$, with Lipschitz constant not larger than 1. For $B \in \mathcal{B}, \varepsilon>0$, we define the $\varepsilon$-neighborhood of $B$ in $\mathcal{P}$

$$
N(B, \varepsilon):=\left\{\left(\sigma_{1}, \sigma_{2}+\delta\right) \in \mathbb{R}^{+} \times \mathbb{R}\left|\left(\sigma_{1}, \sigma_{2}\right) \in B,\right| \delta \mid \leq \varepsilon\right\} .
$$

Theorem 2.4. Let $\mu$ be a finite signed Borel measure on $\mathcal{P}$. Let us suppose that there exists $L>0$ such that

$$
|\mu|(N(B, \varepsilon)) \leq L \varepsilon \quad \forall \varepsilon>0, \forall B \in \mathcal{B},
$$

where $|\mu|$ is the total variation of $\mu$. Then, for every admissible couple $(u, \xi)$ we have $\mathcal{H}_{\mu}[u, \xi] \in C^{0}([0, T])$, and there exists $C>0$ (depending only on $L$ ) such that for every admissible couples $(u, \xi),(v, \eta)$

$$
\left\|\mathcal{H}_{\mu}[u, \xi]-\mathcal{H}_{\mu}[v, \eta]\right\|_{C^{0}([0, T])} \leq C\left(\|u-v\|_{C^{0}([0, T])}+\|\xi-\eta\|_{L^{1}(\mathcal{P},|\mu|)}\right) .
$$

For the proof see Visintin [21] pages 114 and 115, after slight modifications due to our definition of admissible couples. For the proof of the following theorem see Visintin [21] (p. 100).

Theorem 2.5. For any signed Borel measure $\mu$ over $\mathcal{P}$, the corresponding Preisach operator $\mathcal{H}_{\mu}$ fulfills the following semigroup property

$$
\text { if }\left[t_{1}, t_{2}\right] \subset[0, T] \text {, then } \mathcal{H}_{\mu}[u, \xi]\left(t_{2}\right)=\mathcal{H}_{\mu}\left[u\left(t_{1}+\cdot\right), \xi_{t_{1}}\right]\left(t_{2}-t_{1}\right) \text { for every admissible }(u, \xi) \text {. }
$$

Remark 2.6. Let $\nu$ be a finite positive Borel measure on $\mathcal{P}$. If

$$
|\mu|(B) \leq \nu(B) \quad \forall \text { Borel set } B \subseteq \mathcal{P},
$$

then $\mathcal{H}_{\mu}$ is also Lipschitz continuous when $\mathcal{S}$ has the topology of $L^{1}(\mathcal{P}, \nu)$.

Let $(y, \xi) \in \mathcal{O}$. It is important to know how $B_{\xi}$ is linked to the line $\rho_{1}=\rho_{2}$ : by a horizontal segment or by a vertical segment or in a "oblique" manner (the latter may hold only at the initial time). We define

$$
\hat{\rho}_{1}(y, \xi):=\inf \left\{\rho_{1} \leq y \mid\left[\rho_{1}, y\right] \times\{y\} \subset B_{\xi}\right\}, \quad \hat{\rho}_{2}(y, \xi):=\sup \left\{\rho_{2} \geq y \mid\{y\} \times\left[y, \rho_{2}\right] \subset B_{\xi}\right\} .
$$

For instance, when $\hat{\rho}_{1}(y, \xi)<y$, then $B_{\xi}$ is linked by a horizontal segment. At least one from $\hat{\rho}_{1}(y, \xi), \hat{\rho}_{2}(y, \xi)$ always coincides with $y$.

Remark 2.7. When we regard $\mathcal{S}$ as equipped with the topology of $L^{p}(\mathcal{P}, \nu)$, we have to identify functions which coincide $\nu$-a.e. on $\mathcal{P}$. A more rigorous definition of $\mathcal{O}$ should be: $(y, \xi) \in \mathbb{R} \times L^{p}(\mathcal{P}, \nu)$ belongs to $\mathcal{O}$ if and only if there exists a maximal antimonotone graph $B$ on $\overline{\mathcal{P}}$, such that $(y, y) \in B$ and $\xi=1 \nu$-a.e. below $B$, 
and $\xi=-1 \nu$-a.e. above $B$. Hence, for any $(y, \xi) \in \mathcal{O} \subset \mathbb{R} \times L^{p}(\mathcal{P}, \nu)$, we may have different choices of the representative element of the equivalence class, of the graph $B_{\xi}$, and consequently of the quantities $\hat{\rho}_{1}(y, \xi)$ and $\hat{\rho}_{2}(y, \xi)$. However, this will be not a problem for the sequel of the paper: we can just work with an arbitrary choice of the representatives (see also Rem. 4.2). Note that $B_{\xi}, \hat{\rho}_{1}$ and $\hat{\rho}_{2}$ are uniquely defined if $\mathcal{S}$ is equipped with the norm of $L^{p}\left(\mathcal{P}, \mu^{\prime}\right)$, with $\mu^{\prime}$ satisfying

$$
\mu^{\prime}(U)>0 \quad \forall U \subset \mathcal{P} \text { open. }
$$

However, even in that case, $\hat{\rho}_{1}(y, \xi)$ and $\hat{\rho}_{2}(y, \xi)$ are not continuous. Indeed, we can approximate any couple $(y, \xi)$ satisfying $y-\hat{\rho}_{1}(y, \xi)>0$ by couples $\left(y_{n}, \xi_{n}\right)$ satisfying $y_{n}-\hat{\rho}_{1}\left(y_{n}, \xi_{n}\right) \equiv 0$.

Proposition 2.8. Let $\nu$ be a finite positive Borel measure on $\mathcal{P}$. Then the closure of any bounded subset of $\mathcal{O} \subset \mathbb{R} \times L^{2}(\mathcal{P}, \nu)$ is compact.

Proof. First of all note that a subset of $\mathcal{O}$ is bounded if and only if its projection on the $y$-component is bounded in $\mathbb{R}$. Let $\left(y_{n}, \xi_{n}\right)$ be a bounded sequence in $\mathcal{O}$. The boundedness of $y_{n}$ implies that, on every compact set of $\mathcal{P}$, the graphs $B_{\xi_{n}}$ are equibounded and equilipschitzean with respect to the coordinates $\sigma_{1}, \sigma_{2}$. Note that the uniform convergence of $B_{\xi_{n}}$ on a compact set $K \subset \mathcal{P}$ implies that the limit $B$ is still a maximal antimonotone graph on $K$, and that if $\xi$ is the corresponding function on $K$ (i.e. $B=B_{\xi}$ on $K$ ), then $\xi_{n} \rightarrow \xi$ in $L^{2}(K, \nu)$. Hence we obtain the conclusion by the Ascoli-Arzelà Theorem and the fact that $\nu$ is finite on $\mathcal{P}$.

\section{The CONTRol PROBlem}

We consider the following controlled dynamical system

$$
\begin{cases}y^{\prime}(t)=f(y(t), w(t), \alpha(t)) & t>0 \\ w(t)=\mathcal{H}_{\mu}\left[y, \xi_{0}\right](t) & t \geq 0 \\ y(0)=y_{0} & \\ \left(y_{0}, \xi_{0}\right) \in \mathcal{O} & \end{cases}
$$

where the continuous function $f: \mathbb{R} \times \mathbb{R} \times A \rightarrow \mathbb{R}$, with $A \subset \mathbb{R}^{q}$ compact, satisfies

$$
\begin{aligned}
& \exists M>0 \text { such that }|f(y, w, a)| \leq M \forall(y, w, a) \in \mathbb{R} \times \mathbb{R} \times A \\
& \left|f\left(y_{1}, w_{1}, a\right)-f\left(y_{2}, w_{2}, a\right)\right| \leq M\left(\left|y_{1}-y_{2}\right|+\left|w_{1}-w_{2}\right|\right) \forall\left(y_{1}, w_{1}, a\right),\left(y_{2}, w_{2}, a\right) \in \mathbb{R} \times \mathbb{R} \times A ;
\end{aligned}
$$

and $\alpha$ is a measurable control, that is

$$
\alpha \in \mathcal{A}:=\{\beta:[0,+\infty[\rightarrow A \text { measurable }\} .
$$

The following result follows from the Lipschitz continuity of $f$, and from the Lipschitz continuity and the semigroup property of the Preisach operator (Ths. 2.4 and 2.5, see also Rem. 2.6). The regularity of the output $w$ comes from some regularity results of the Preisach operator (see Visintin [21]; however, we only need continuity of $w$ ). The proof of the existence can be given by the contraction principle or by a delayed approximation argument; the estimate (3.3) can be proved using also the Gronwall inequality (see for instance Brokate-Sprekels [9] and Bagagiolo [2]).

Proposition 3.1. Let $\mu$ and $\nu$ be respectively a finite signed Borel measure and a finite positive Borel measure on $\mathcal{P}$ satisfying (2.2) and (2.5). For any choice of $\left(y_{0}, \xi_{0}\right) \in \mathcal{O}$ and $\alpha \in \mathcal{A}$, there exists a unique solution $(y(\cdot), w(\cdot))$ of the system (3.1), defined in $\left[0,+\infty\left[\right.\right.$. Moreover, we also denote by $t \mapsto \xi_{t}$ the evolution of the internal variables $\xi$. It is easy to see that $\left(y(t), \xi_{t}\right) \in \mathcal{O}$ for every $t \geq 0$. Moreover, we have the following regularities: $y(\cdot) \in W_{\text {loc }}^{1,1}\left(0,+\infty[, \mathbb{R}), w(\cdot) \in W_{\text {loc }}^{1,1}\left(0,+\infty[, \mathbb{R})\right.\right.$, and $\xi_{(\cdot)} \in C\left(\left[0,+\infty\left[, L^{1}(\mathcal{P}, \nu)\right)\right.\right.$. Finally, for every $T>0$ there exists a modulus of continuity $\omega_{T}$ such that, for every initial values $\left(y_{1}, \xi_{1}\right),\left(y_{2}, \xi_{2}\right) \in \mathcal{O}$, and 
for every control $\alpha \in \mathcal{A}$ the following inequality holds (here $\left(y_{i}(\cdot), w_{i}(\cdot)\right)$ is the solution corresponding to $\left(y_{i}, \xi_{i}\right)$ as initial datum, and $\alpha$ as control)

$$
\left|y_{1}(t)-y_{2}(t)\right|+\left|w_{1}(t)-w_{2}(t)\right| \leq \omega_{T}\left(\max \left\{\left|y_{1}-y_{2}\right|,\left\|\xi_{1}-\xi_{2}\right\|_{L^{1}(\mathcal{P}, \nu)}\right\}\right) \forall t \in[0, T] .
$$

Remark 3.2. Since $\left\|\xi_{1}-\xi_{2}\right\|_{L^{1}(\mathcal{P}, \nu)}=\left\|\xi_{1}-\xi_{2}\right\|_{L^{2}(\mathcal{P}, \nu)}^{2} / 2$ for every $\xi_{1}, \xi_{2} \in \mathcal{S}$, then the trajectory is also continuous for the topology of $L^{2}(\mathcal{P}, \nu)$.

Remark 3.3. In general, the evolution of the internal variables $t \rightarrow \xi_{t}$ does not belong to $W^{1,1}\left(0, T ; L^{2}(\mathcal{P}, \nu)\right)$, even if the input $u$ is very regular. Indeed, let us suppose that the input is increasing and that we start from an initial configuration $\xi_{0}$ which corresponds to a maximal antimonotone graph with horizontal final segment (for instance the situation in Fig. 2ii). To simplify the calculation, let us suppose that $\nu$ locally around the horizontal segment coincides with the Lebesgue measure. We take a function $\psi \in C^{0}(\mathcal{P})$, and a function $\varphi \in C_{c}^{\infty}(0, T)$. Then, the function $t \rightarrow \varphi(t) \psi(\cdot)$ belongs to $C_{c}^{\infty}\left(0, T ; L^{2}(\mathcal{P}, \nu)\right)$. Let us call $Q_{t}$ the region of the Preisach plane that, at time $t$, is delimited by the two graphs $B_{\xi_{0}}$ and $B_{\xi_{t}}$ : it is the "trapezoidal" region where we had the switching from -1 to +1 (compare with Fig. 3 for a similar situation). Hence, we have (recall that the input $u$ is increasing) $\xi_{t}=\xi_{0}+2 \chi_{Q_{t}}$, where $\chi_{A}$ is the characteristic function of the set $A$. Hence we get

$$
\int_{0}^{T} \int_{\mathcal{P}} \xi_{t}(\rho) \dot{\varphi}(t) \psi(\rho) \mathrm{d} \rho \mathrm{d} t=2 \int_{0}^{T} \dot{\varphi}(t) \int_{Q_{t}} \psi(\rho) \mathrm{d} \rho \mathrm{d} t .
$$

Denoting by $g(t)$ the function of time $t \rightarrow \int_{Q_{t}} \psi \mathrm{d} \rho$, it is not hard to check that $g \in W^{1,1}(0, T)$ and that its weak derivative is $\dot{g}(t)=\int_{u(t)}^{\hat{\rho}_{1}\left(u(t), \xi_{t}\right)} \psi\left(\rho_{1}, u(t)\right) d \rho_{1}$. Hence, integrating by parts in (3.4), we get

$$
-2 \int_{0}^{T} \varphi(t) \int_{u(t)}^{\hat{\rho}_{1}\left(u(t), \xi_{t}\right)} \psi\left(\rho_{1}, u(t)\right) \mathrm{d} \rho_{1}
$$

which shows that the weak derivative of $t \rightarrow \xi_{t}$ involves the evaluation on the horizontal segment, and hence it is not a function.

Let us consider a nonnegative function (running cost)

$$
\ell: \mathbb{R} \times \mathbb{R} \times A \rightarrow[0,+\infty[\text { continuous }
$$

such that for some $M>0$ and a some modulus of continuity $\omega$ it satisfies, $\forall(y, w, a),\left(y_{1}, w_{1}, a\right),\left(y_{2}, w_{2}, a\right) \in$ $\mathbb{R} \times \mathbb{R} \times A$,

$$
|\ell(y, w, a)| \leq M, \quad\left|\ell\left(y_{1}, w_{1}, a\right)-\ell\left(y_{1}, w_{1}, a\right)\right| \leq \omega\left(\left|y_{1}-y_{2}\right|+\left|w_{1}-w_{2}\right|\right) .
$$

Moreover let us consider a nonnegative function (final cost)

$$
h: \mathbb{R} \times \mathbb{R} \rightarrow[0,+\infty[\text { bounded and uniformly continuous. }
$$

Finally, let $\lambda \geq 0$ be a discount factor. For any initial state $(y, \xi) \in \mathcal{O}$, any control $\alpha \in \mathcal{A}$, and any time $t \geq 0$, we consider the following cost functional

$$
J(y, \xi, t, \alpha):=\int_{0}^{t} \mathrm{e}^{-\lambda s} \ell(y(s), w(s), \alpha(s)) \mathrm{d} s+\mathrm{e}^{-\lambda t} h(y(t), w(t)),
$$

where $(y(\cdot), w(\cdot))$ is the trajectory of $(3.1)$ with initial state $(y, \xi)$ and control $\alpha$. The value function is

$$
V(y, \xi, t):=\inf _{\alpha \in \mathcal{A}} J(y, \xi, t, \alpha) \quad \forall(y, \xi, t) \in \mathcal{O} \times[0,+\infty[.
$$




\section{Dynamic PRogramming}

Due to the semigroup property (2.4) of the Preisach operator, the Dynamic Programming Principle holds.

Theorem 4.1 (DPP: Dynamic Programming Principle). For all $(y, \xi) \in \mathcal{O}$, for all $t \geq 0$ and for all $0 \leq \tau \leq t$

$$
V(y, \xi, t)=\inf _{\alpha \in \mathcal{A}}\left(\int_{0}^{\tau} \mathrm{e}^{-\lambda s} \ell(y(s), w(s), \alpha(s)) \mathrm{d} s+\mathrm{e}^{-\lambda \tau} V\left(y(\tau), \xi_{\tau}, t-\tau\right)\right) .
$$

\subsection{Test functions}

In this subsection, $\nu$ is a finite positive Borel measure on $\mathcal{P}$. For every $s>0$ and for every $(y, \xi) \in \mathcal{O}$ we define the subsets of $\mathcal{P}$

$$
\begin{aligned}
& \mathcal{P}_{s}^{u}(y, \xi):=\left\{\rho \in \mathcal{P} \mid \hat{\rho}_{1}(y, \xi) \leq \rho_{1} \leq y, y \leq \rho_{2} \leq y+s\right\} \\
& \mathcal{P}_{s}^{l}(y, \xi):=\left\{\rho \in \mathcal{P} \mid y-s \leq \rho_{1} \leq y, y \leq \rho_{2} \leq \hat{\rho}_{2}(y, \xi)\right\}
\end{aligned}
$$

The superscripts $u$ and $l$ respectively stay for "upper" and "left".

Let us consider a function $\phi \in C^{2}\left(L^{2}(\mathcal{P}, \nu)\right)$. We identify $L^{2}(\mathcal{P}, \nu)$ with its dual and denote by $D \phi(\xi)$ and by $D^{2} \phi(\xi)$ respectively the Fréchet differential and the second Fréchet differential of $\phi$ in $\xi$. In particular, $D \phi(\xi)$ represents the linear form on $L^{2}(\mathcal{P}, \nu)$ acting as

$$
\langle D \phi(\xi), \psi\rangle=\int_{\mathcal{P}} D \phi(\xi) \psi \mathrm{d} \nu,
$$

and $D^{2} \phi(\xi)$ is a bounded symmetric bilinear form on $L^{2}(\mathcal{P}, \nu)$, whose value on the couple $(\psi, \eta)$ we write as

$$
\left[D^{2} \phi(\xi)\right](\psi, \eta)
$$

Let $\mathcal{L}$ be the space of the symmetric bounded bilinear forms on $L^{2}(\mathcal{P}, \nu)$. For $(y, \xi) \in \mathcal{O},(\psi, \pi) \in L^{2}(\mathcal{P}, \nu) \times \mathcal{L}$, let $T^{u}[\psi, \pi](y, \xi)$ and $T^{l}[\psi, \pi](y, \xi)$ be respectively the following limits (which may possibly not exist)

$$
\begin{aligned}
T^{u}[\psi, \pi](y, \xi): & =\lim _{s \rightarrow 0^{+}} \frac{1}{s}\left(2\left\langle\psi, \chi_{\mathcal{P}_{s}^{u}(y, \xi)}\right\rangle+2 \pi\left(\chi_{\mathcal{P}_{s}^{u}(y, \xi)}, \chi_{\mathcal{P}_{s}^{u}(y, \xi)}\right)\right) \\
T^{l}[\psi, \pi](y, \xi): & =\lim _{s \rightarrow 0^{+}} \frac{1}{s}\left(2\left\langle\psi, \chi_{\mathcal{P}_{s}^{l}(y, \xi)}\right\rangle-2 \pi\left(\chi_{\mathcal{P}_{s}^{l}(y, \xi)}, \chi_{\mathcal{P}_{s}^{l}(y, \xi)}\right)\right)
\end{aligned}
$$

where $\chi_{C}$ is the characteristic function of $C \subset \mathcal{P}$.

Remark 4.2. It is not hard to check that the definitions of $T^{u}$ and $T^{l}$ are independent from the particular representative of the equivalence class of $(y, \xi) \in \mathcal{O} \subset \mathbb{R} \times L^{2}(\mathcal{P}, \nu)$, and from the corresponding graph $B_{\xi}$ (see also Rem. 2.7).

We define the following set of test functions

$\mathcal{V}:=\left\{\phi \in C^{2}\left(L^{2}(\mathcal{P}, \nu)\right)\right.$ such that $D \phi(\xi) \in L^{\infty}(\mathcal{P}, \nu) \forall \xi \in \mathcal{S}$, and

$$
\left.T^{u}\left[D \phi(\xi), D^{2} \phi(\xi)\right](y, \xi), T^{l}\left[D \phi(\xi), D^{2} \phi(\xi)\right](y, \xi) \text { exist } \forall(y, \xi) \in \mathcal{O}\right\}
$$

Under fairly general hypotheses, the set $\mathcal{V}$ is certainly not empty. Indeed, let us suppose that $\nu$ is absolutely continuous with respect to the Lebesgue measure, and that its density $g(\rho)($ i.e. $\mathrm{d} \nu(\rho)=g(\rho) \mathrm{d} \rho)$ is continuous (we need much less, but note that after the condition (2.5), the choice of $\nu$ is at our disposal). On every 
horizontal and vertical segment of the form $[a, b] \times\{y\}$ and $\{y\} \times[c, d]$, we then have respectively the measures $\mathrm{d} \nu_{1}\left(\rho_{1}\right):=g\left(\rho_{1}, y\right) \mathrm{d} \rho_{1}$ and $\mathrm{d} \nu_{2}\left(\rho_{2}\right):=g\left(y, \rho_{2}\right) \mathrm{d} \rho_{2}$ (dropping in the entry of $\nu_{i}$ the indication of $y$, which may be obvious by the context). Moreover, for every $\xi \in \mathcal{S}$, and for every horizontal and vertical segment $[a, b] \times\{y\}$ and $\{y\} \times[c, d]$, let us define the "up-trace" $\xi_{u} \in L^{2}\left(a, b ; \nu_{1}\right)$ and the "left-trace" $\xi_{l} \in L^{2}\left(c, d ; \nu_{2}\right)$ respectively as the traces on $[a, b] \times\{y\}$ and on $\{y\} \times[c, d]$ of $\xi$ regarded as function on the rectangles $(a, b) \times(y, y+s)$ and $(y-s, y) \times(c, d)$ with $s>0$. Note that, by the definition of $\mathcal{S}$, such traces are well defined, and they are independent on the equivalence class of $\xi$ in $L^{2}(\mathcal{P}, \nu)$. Now, let us take $(\bar{y}, \bar{\xi}) \in \mathcal{O}$, and consider the function

$$
\phi: \xi \mapsto\|\xi-\bar{\xi}\|_{L^{2}(\mathcal{P}, \nu)}^{2} .
$$

For every $(y, \xi) \in \mathcal{O}$, we have (note that on $\mathcal{P}_{\tau}^{u}(y, \xi)$ it is $\xi \equiv-1$ )

$$
\begin{aligned}
T^{u}\left[D \phi(\xi), D^{2} \phi(\xi)\right](y, \xi) & =\lim _{s \rightarrow 0} \frac{1}{s}\left(2\left\langle D \phi(\xi), \chi_{\mathcal{P}_{s}^{u}(y, \xi)}\right\rangle+2\left[D^{2} \phi(\xi)\right]\left(\chi_{\mathcal{P}_{s}^{u}(y, \xi)}, \chi_{\mathcal{P}_{s}^{u}(y, \xi)}\right)\right) \\
& =\lim _{s \rightarrow 0} \frac{4}{s} \int_{\mathcal{P}_{s}^{u}(y, \xi)}(\xi-\bar{\xi}+1) \mathrm{d} \nu=\lim _{s \rightarrow 0}-\frac{4}{s} \int_{\mathcal{P}_{s}^{u}(y, \xi)} \bar{\xi} \mathrm{d} \nu=-4 \int_{\hat{\rho}_{1}(y, \xi)}^{y} \bar{\xi}_{u}\left(\rho_{1}, y\right) \mathrm{d} \nu_{1}\left(\rho_{1}\right) .
\end{aligned}
$$

In the same way we have

$$
T^{l}\left[D \phi(\xi), D^{2} \phi(\xi)\right](y, \xi)=-4 \int_{y}^{\hat{\rho}_{2}(y, \xi)} \bar{\xi}_{l}\left(y, \rho_{2}\right) \mathrm{d} \nu_{2}\left(\rho_{2}\right) .
$$

Hence, when $\bar{\xi} \in \mathcal{S}$, the function (4.4) belongs to $\mathcal{V}$. There are other examples of functions in $\mathcal{V}$, which will be useful in the sequel. In a similar way as before, we can see that for every open regular subset $C \subset \mathcal{P}$, considering the measure $\mathrm{d} \nu_{C}:=\chi_{C} \mathrm{~d} \nu$ on $\mathcal{P}$, the function

$$
\varphi: \xi \mapsto\|\xi-\bar{\xi}\|_{L^{2}\left(\mathcal{P}, \nu_{C}\right)}^{2}
$$

belongs to $\mathcal{V}$. Finally, the following function also belongs to $\mathcal{V}$

$$
\phi: \xi \mapsto \mathrm{e}^{\int_{\mathcal{P}} \xi \mathrm{d} \nu_{C}} .
$$

\subsection{The Hamilton-Jacobi equation}

We will use the following hypothesis on the Borel finite measures $\mu$ (signed) and $\nu$ (positive) on $\mathcal{P}\left(\mathcal{L}^{2}\right.$ is the Lebesgue measure on $\mathcal{P}$ )

$\nu$ has density $g \in L^{\infty}\left(\mathcal{P}, \mathcal{L}^{2}\right)$ with respect to $\mathcal{L}^{2} ;$ the functions (4.4)-(4.7) belong to $\mathcal{V} ;(2.2),(2.5)$ hold.

We will always consider $\mathcal{O}$ equipped with the topology of $\mathbb{R} \times L^{2}(\mathcal{P}, \nu)$.

Remark 4.3. The hypothesis (4.8) certainly holds if, for instance, the measure $\mu$ has density with respect to the Lebesgue measure which also belongs to $L^{\infty}\left(\mathcal{P}, \mathcal{L}^{2}\right)$. This requirement is always satisfied in the engineering applications of the Preisach operator, see Mayergoyz [18] and Della Torre [11].

We consider the following infinite dimensional Hamiltonian, defined on a subset of $\mathcal{O} \times \mathbb{R} \times L^{2}(\mathcal{P}, \nu) \times \mathcal{L}$

$$
H(y, \xi, p, \psi, \pi):=\sup _{a \in A}\left\{-p f(y, w, a)-\left(f^{+}(y, w, a) T^{u}[\psi, \pi](y, \xi)-f^{-}(y, w, a) T^{l}[\psi, \pi](y, \xi)\right)-\ell(y, w, a)\right\},
$$

where $f^{+}=\max (0, f), f^{-}=\max (0,-f)$ and $w=\int_{\mathcal{P}} \xi \mathrm{d} \mu$. 
The hamiltonian (4.9) is not continuous for the topology of $\mathcal{O}$ (see Rem. 2.7). Hence, in order to perform viscosity solution techniques, we consider the following "lower" and "upper" hamiltonians $H^{-}$and $H^{+}$:

$$
\begin{aligned}
& H^{-}(y, \xi, p, \psi, \pi):= \\
& \quad \sup _{a \in A}\left\{-p f(y, w, a)-\left(f^{+}(y, w, a)\left(T^{u}[\psi, \pi](y, \xi)\right)^{+}+f^{-}(y, w, a)\left(T^{l}[\psi, \pi](y, \xi)\right)^{-}\right)-\ell(y, w, a)\right\}, \\
& H^{+}(y, \xi, p, \psi, \pi):= \\
& \quad \sup _{a \in A}\left\{-p f(y, w, a)+\left(f^{+}(y, w, a)\left(T^{u}[\psi, \pi](y, \xi)\right)^{-}+f^{-}(y, w, a)\left(T^{l}[\psi, \pi](y, \xi)\right)^{+}\right)-\ell(y, w, a)\right\} .
\end{aligned}
$$

In the sequel $B U C(\mathcal{O} \times[0, T])$ is the space of bounded and uniformly continuous functions on $\mathcal{O} \times[0, T] ; V_{t}$ and $V_{y}$ mean derivatives, and $D$ is the differential with respect to $\xi \in L^{2}(\mathcal{P}, \nu)$.

Theorem 4.4. Let (4.8) hold. For every $T>0$ the following holds: the value function $V$ belongs to $B U C(\mathcal{O} \times$ $[0, T])$; moreover it is a viscosity solution of the following Cauchy problem for a Hamilton-Jacobi equation

$$
\left\{\begin{array}{l}
\left.\left.V_{t}(y, \xi, t)+\lambda V(y, \xi, t)+H\left(y, \xi, V_{y}(y, \xi, t), D V(y, \xi, t), D^{2} V(y, \xi, t)\right)=0 \quad \text { in } \mathcal{O} \times\right] 0, T\right] \\
V(y, \xi, 0)=h(y, w)
\end{array}\right.
$$

with test functions taken in $\left.\left.C^{1}(\mathbb{R} \times] 0, T\right]\right) \times \mathcal{V}$, and $w=\int_{\mathcal{P}} \xi \mathrm{d} \mu$.

By viscosity solution of $(4.12)$ with $\left.\left.C^{1}(\mathbb{R} \times] 0, T\right]\right) \times \mathcal{V}$ as space of test functions we mean that $V$ satisfies the initial condition and that:

Subsolution. For every $(\bar{y}, \bar{\xi}, \bar{t}) \in \mathcal{O} \times] 0, T]$, every $\left.\left.\varphi \in C^{1}(\mathbb{R} \times] 0, T\right]\right)$, and every $\phi \in \mathcal{V}$ such that $(\bar{y}, \bar{\xi}, \bar{t})$ is a local maximum for $V(y, \xi, t)-\varphi(y, t)-\phi(\xi)$ with respect to $\mathcal{O} \times] 0, T]$, the following holds

$$
\varphi_{t}(\bar{y}, \bar{t})+\lambda V(\bar{y}, \bar{\xi}, \bar{t})+H^{-}\left(\bar{y}, \bar{\xi}, \varphi_{y}(\bar{y}, \bar{t}), D \phi(\bar{\xi}), D^{2} \phi(\bar{\xi})\right) \leq 0
$$

Supersolution. For every $(\bar{y}, \bar{\xi}, \bar{t}) \in \mathcal{O} \times] 0, T]$, every $\left.\left.\varphi \in C^{1}(\mathbb{R} \times] 0, T\right]\right)$, and every $\phi \in \mathcal{V}$ such that $(\bar{y}, \bar{\xi}, \bar{t})$ is a local minimum for $V(y, \xi, t)-\varphi(y, t)-\phi(\xi)$ with respect to $\mathcal{O} \times] 0, T]$, the following holds

$$
\varphi_{t}(\bar{y}, \bar{t})+\lambda V(\bar{y}, \bar{\xi}, \bar{t})+H^{+}\left(\bar{y}, \bar{\xi}, \varphi_{y}(\bar{y}, \bar{t}), D \phi(\bar{\xi}), D^{2} \phi(\bar{\xi})\right) \geq 0
$$

Proof. (Th. 4.4.) The fact that $V \in B U C(\mathcal{O} \times[0, T])$ comes from (3.2)-(3.6) and Remark 3.2 (see Bardi-Capuzzo Dolcetta [4] page 148 and Bagagiolo [2]).

By the definition, it is evident that $V$ satisfies the initial condition.

Let us prove that $V$ is subsolution. Since the equation consists of a "finite dimensional part" and of an "infinite dimensional part" (i.e. the part involving $T^{u}$ and $T^{l}$ ), and since the technique we are going to use is standard for the finite dimensional one, we will be mostly concerned with the treatment of the infinite dimensional part, referring the reader to Bardi-Capuzzo Dolcetta [4] for other details.

We prove that $V$ satisfies (4.13) even replacing $H^{-}$by $H$ itself, which a fortiori will give the conclusion. Let us take $\left.\left.(y, \xi, t) \in \mathcal{O} \times] 0, T], \varphi \in C^{1}(\mathbb{R} \times] 0, T\right]\right)$ and $\phi \in \mathcal{V}$ such that $V-\varphi-\phi$ has a local maximum in $(y, \xi, t)$ with respect to $\mathcal{O} \times] 0, T]$. Recall that $\mathcal{O}$ is an invariant set for the trajectory $\left(y(t), \xi_{t}\right)$ given by the solution of the system (3.1). Fix any constant control $\alpha \equiv a \in A$, write $w=\int_{\mathcal{P}} \xi \mathrm{d} \mu$ and consider the evolutions $y(\cdot), w(\cdot)$, $\xi_{(\cdot)}$ given by the system (3.1), with $\alpha$ as control, and $(y, \xi)$ as initial datum. For $\delta>0$ sufficiently small, for every $0 \leq \tau \leq \delta \leq t$ we have

$$
\varphi(y, t)+\phi(\xi)-\varphi(y(\tau), t-\tau)-\phi\left(\xi_{\tau}\right) \leq V(y, \xi, t)-V\left(y(\tau), \xi_{\tau}, t-\tau\right) .
$$




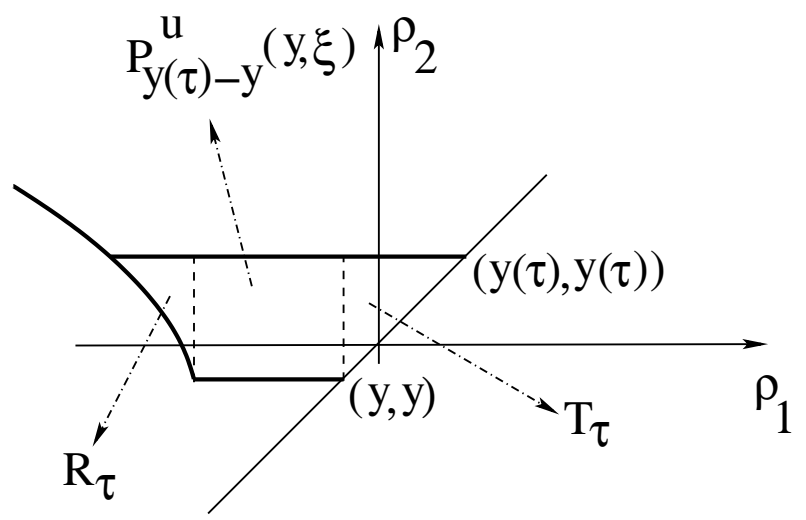

FiguRE 3. Graphic explanation for the proof of Theorem 4.4.

By DPP (4.1), we get

$$
\varphi(y, t)+\phi(\xi)-\varphi(y(\tau), t-\tau)-\phi\left(\xi_{\tau}\right) \leq \int_{0}^{\tau} \mathrm{e}^{-\lambda s} \ell(y(s), w(s), a) \mathrm{d} s+V\left(y(\tau), \xi_{\tau}, t-\tau\right)\left(\mathrm{e}^{-\lambda \tau}-1\right)
$$

We divide (4.15) by $\tau$ and let $\tau \rightarrow 0^{+}$. Since $0<t-\tau<T$, by the regularity of $\varphi$, even if $t=T$, we can perform such limit. Since the evolution of the internal variables is not differentiable (see Rem. 3.3), we cannot apply the chain rule to the difference $\phi(\xi)-\phi\left(\xi_{\tau}\right)$. We have three possibilities:

a) $f(y, w, a)=0$. Then $y(\cdot) \equiv y, \xi_{(\cdot)} \equiv \xi, w(\cdot) \equiv w$ and we get

$$
\varphi_{t}(y, t)+\lambda V(y, \xi, t)-\ell(y, w, a) \leq 0
$$

b) $f(y, w, a)>0$. For small $\delta>0, y(\cdot)$ is increasing in $[0, \delta]$. Hence, see Figure 3 , in the Preisach plane $\mathcal{P}$ the horizontal segment $\left[\hat{\rho}_{1}\left(y(\tau), \xi_{\tau}\right), y(\tau)\right] \times\{y(\tau)\}$ is moving up, starting from the possibly degenerate (that is a point) initial one $\left[\hat{\rho}_{1}(y, \xi), y\right] \times\{y\}$. Hence, $\hat{\rho}_{2}\left(y(\tau), \xi_{\tau}\right)=y(\tau)$ for every $0<\tau \leq \delta$, and $\tau \rightarrow \hat{\rho}_{1}\left(y(\tau), \xi_{\tau}\right)$ is non increasing and right-continuous in $\tau=0$.

We claim that

$$
\xi_{\tau}-\xi=2 \chi_{\mathcal{P}_{f(y, w, a) \tau}^{u}}(y, \xi)+\sigma(\tau)
$$

where $\sigma(\tau)$ is a function $\eta_{\tau}$ such that $\left\|\eta_{\tau}\right\|_{L^{1}(\mathcal{P}, \nu)} / \tau \rightarrow 0$ as $\tau \rightarrow 0$. Indeed, $\xi_{\tau}-\xi=2 \chi_{\mathcal{P}(\tau)}$ where $\mathcal{P}(\tau)$ is a "trapezoidal" region given by the disjoint union of the following sets:

i) the rectangle $\mathcal{P}_{y(\tau)-y}^{u}(y, \xi)$ (which may be reduced to a vertical segment if $\left.\hat{\rho}_{1}(y, \xi)=y\right)$; ii) the triangle $T_{\tau}$ with vertices $(y, y),(y, y(\tau)),(y(\tau), y(\tau))$; iii) the "triangular region" $R_{\tau}=\left\{\left(\rho_{1}, \rho_{2}\right) \in \mathcal{P} \mid \rho_{1} \leq \hat{\rho}_{1}(y, \xi), B_{\xi}\left(\rho_{1}\right) \leq\right.$ $\left.\rho_{2} \leq y(\tau)\right\}$ (which, when $B_{\xi}$ is linked to the line $\rho_{1}=\rho_{2}$ by a vertical segment or when $\hat{\rho}_{1}(y, \xi)=-\infty$, at least for small $\tau$, may be empty). Note that $\nu\left(T_{\tau}\right)=o(\tau)$ and $\nu\left(\mathcal{P}_{y(\tau)-y}^{u}(y, \xi)\right)=O(\tau)$. We have also $\nu\left(R_{\tau}\right)=o(\tau)$, since $\lim _{\tau \rightarrow 0^{+}} \hat{\rho}_{1}\left(y(\tau), \xi_{\tau}\right)=\hat{\rho}_{1}(y, \xi)$ (see also (4.8)). Finally, since $y(\tau)=y+f(y, w, a) \tau+o(\tau)$, we get

$$
\chi_{\mathcal{P}_{y(\tau)-y}^{u}}(y, \xi)=\chi_{\mathcal{P}_{f(y, w, a) \tau}^{u}(y, \xi)}+\sigma(\tau)
$$


and the claim is proven. Hence, by (4.17), using the Taylor's second order formula for $\phi$ in $\xi$, and recalling that $D \phi(\xi) \in L^{\infty}(\mathcal{P}, \nu)$, we conclude that

$$
\begin{aligned}
\lim _{\tau \rightarrow 0^{+}} \frac{\phi\left(\xi_{\tau}\right)-\phi(\xi)}{\tau} & =f(y, w, a) \lim _{\tau \rightarrow 0^{+}} \frac{2\left\langle D \phi(\xi), \chi_{\mathcal{P}_{f(y, w, a) \tau}^{u}}\right\rangle+2\left[D^{2} \phi(\xi)\right]\left(\chi_{\mathcal{P}_{f(y, w, a) \tau}^{u}}^{u}, \chi_{\mathcal{P}_{f(y, w, a) \tau}^{u}}^{u}\right)}{f(y, w, a) \tau} \\
& =f(y, w, a) T^{u}\left[D \phi(\xi), D^{2} \phi(\xi)\right](y, \xi),
\end{aligned}
$$

where we dropped the entry $(y, \xi)$ in $\mathcal{P}_{f(y, w, a) \tau}^{u}(y, \xi)$. This concludes b).

Note that we are forced to use the second order Taylor's formula, since we are working in $L^{2}(\mathcal{P}, \nu)$. Indeed, if we stop to the first order, then we get a term of the kind $o\left(\left\|\chi_{\mathcal{P}_{s}^{u}}\right\|_{L^{2}(\mathcal{P}, \nu)}\right) / s$. But in general, we can only say that $\left\|\chi_{\mathcal{P}_{s}^{u}}\right\|_{L^{2}(\mathcal{P}, \nu)}$ goes to zero at least as $\sqrt{s}$;

c) $f(y, w, a)<0$. We argue as in the case b). We have that $y(\cdot)$ is decreasing in $[0, \delta]$ for small $\delta$. Hence, in the Preisach plane $\mathcal{P}$ the vertical segment $\{y(\tau)\} \times\left[y, \hat{\rho}_{2}\left(y(\tau), \xi_{\tau}\right)\right]$ is moving towards left, starting from the possibly degenerate (that is a point) initial one $\{y\} \times\left[y, \hat{\rho}_{2}(y, \xi)\right]$. Hence, $\hat{\rho}_{1}\left(y(\tau), \xi_{\tau}\right)=y(\tau)$ for every $0<\tau \leq \delta$, and $\tau \rightarrow \hat{\rho}_{2}\left(y(\tau), \xi_{\tau}\right)$ non decreasing and right-continuous in $\tau=0$. In a similar way as above, we have that

$$
\xi_{\tau}-\xi=-2 \chi_{\mathcal{P}_{-f(y, w, a) \tau}^{l}(y, \xi)}+\sigma(\tau) .
$$

Hence, we conclude the step c) by (we drop again the entry $(y, \xi)$ )

$$
\begin{aligned}
\lim _{\tau \rightarrow 0^{+}} \frac{\phi\left(\xi_{\tau}\right)-\phi(\xi)}{\tau} & =f(y, w, a) \lim _{\tau \rightarrow 0^{+}} \frac{2\left\langle D \phi(\xi), \chi_{\mathcal{P}_{-} f(y, w, a) \tau}\right\rangle-2\left[D^{2} \phi(\xi)\right]\left(\chi_{\mathcal{P}_{-} l_{f(y, w, a) \tau}}, \chi_{\mathcal{P}_{-} f(y, w, a) \tau}\right)}{-f(y, w, a) \tau} \\
& =f(y, w, a) T^{l}\left[D \phi(\xi), D^{2} \phi(\xi)\right](y, \xi) .
\end{aligned}
$$

By the arbitrariness of $a \in A$, using standard techniques we can conclude.

Now, let us prove that $V$ is supersolution. Let $\left.\left.\phi \in \mathcal{V}, \varphi \in C^{1}(\mathbb{R} \times] 0, T\right]\right)$, be such that $\left.\left.(y, \xi, t) \in \mathcal{O} \times\right] 0, T\right]$ is of local minimum for $V-\varphi-\phi$ with respect to $\mathcal{O} \times] 0 . T]$. For any $\varepsilon>0$ and any $\tau>0$ sufficiently small, arguing as in the step b), by DPP we find a measurable control $\alpha(\cdot)$, depending on $\varepsilon$ and $\tau$, such that

$$
\varphi(y, t)+\phi(\xi)-\varphi(y(\tau), t-\tau)-\phi\left(\xi_{\tau}\right) \geq \int_{0}^{\tau} \mathrm{e}^{-\lambda s} \ell(y(s), w(s), \alpha(s)) \mathrm{d} s+V\left(y(\tau), \xi_{\tau}, t-\tau\right)\left(1-\mathrm{e}^{-\lambda \tau}\right)-\tau \varepsilon
$$

As usual, to prove that the value function is a supersolution is slightly harder. The main reason is that we cannot use constant controls as for the subsolution case. In particular, for what concerns our problem, $y(\cdot)$ may not be monotone. Hence, we use some "memory effects" of the hysteresis relationship in order to recover a monotone case. This will directly lead us to prove (4.14), and not the same inequality replacing $H^{+}$by $H$, which probably is not true.

Let us suppose that $\hat{\rho}_{2}(y, \xi)=y$. For any $\tau$ we define

$$
\begin{aligned}
& y_{+}(\tau):=\max _{s \in[0, \tau]} y(s), \quad \xi_{+}[\tau]:=\text { internal variable of } \mathcal{H}_{\mu}\left[y_{+}, \xi\right](\tau) \\
& y_{-}(\tau):=\min _{s \in[0, \tau]} y(s), \quad \xi_{-}[\tau]:=\text { internal variable of } \mathcal{H}_{\mu}\left[y_{-}, \xi\right](\tau)
\end{aligned}
$$

We claim that, at any instant $\tau \geq 0$, we have ( $\sigma$ is as in (4.17))

$$
\xi_{\tau}=\xi_{+}[\tau]+\sigma(\tau) .
$$

We define $r(\tau):=\max \left(y_{+}(\tau), \hat{\rho}_{2}\left(y_{-}(\tau), \xi_{-}[\tau]\right)\right)$. Since $\hat{\rho}_{2}(y, \xi)=y$, then $\lim _{\tau \rightarrow 0^{+}} \hat{\rho}_{2}\left(y_{-}(\tau), \xi_{-}[\tau]\right)=y$ and hence $\lim _{\tau \rightarrow 0^{+}} r(\tau)=y$. Moreover, $\left|\xi_{\tau}-\xi_{+}[\tau]\right| \leq 2 \chi_{T}$, where $T$ is the triangle of vertices $\left(y_{-}(\tau), y_{-}(\tau)\right)$, 


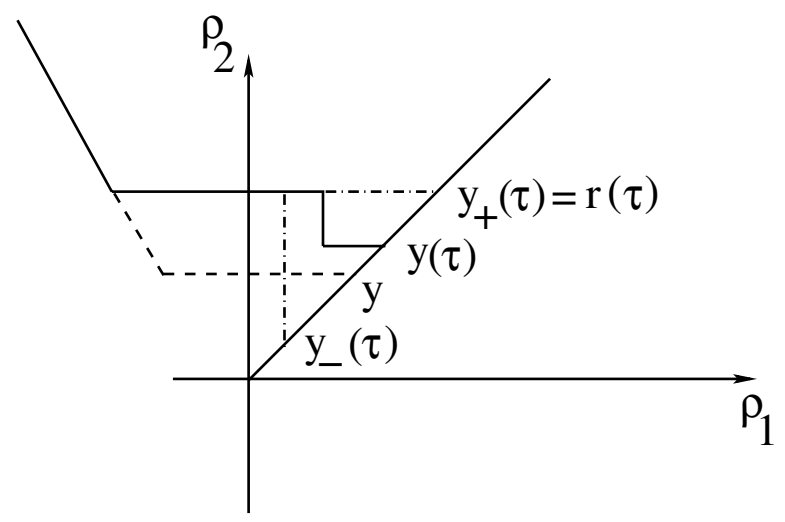

FigurE 4 . The filled graph is $B_{\xi_{\tau}}$; the dashed graph is $B_{\xi}$; the pointed-dashed lines define the triangle $T$ (see the proof of Th. 4.4).

$\left(y_{-}(\tau), r(\tau)\right)$, and $(r(\tau), r(\tau))$ (see the example in Fig. 4). Hence (4.20) follows. We suppose that (see also Lem. 4.5 below), for every $\tau>0$ and $\varepsilon>0$, the following inequality holds

$$
y_{+}(\tau)-y=\int_{0}^{\tau} \chi_{d}\left(y(s), y_{+}(s)\right) f^{+}\left(y_{+}(s), w(s), \alpha(s)\right) \mathrm{d} s>0 .
$$

We use the notations $c_{+}(\tau):=y_{+}(\tau)-y$ and $\chi_{\tau}:=\chi_{\mathcal{P}_{c_{+}(\tau)}^{u}}(y, \xi)$. We get

$$
\begin{aligned}
\frac{\phi\left(\xi_{\tau}\right)-\phi(\xi)}{\tau} & =\frac{c_{+}(\tau)}{\tau}\left(\frac{2\left\langle D \phi(\xi), \chi_{\tau}\right\rangle+2\left[D^{2} \phi(\xi)\right]\left(\chi_{\tau}, \chi_{\tau}\right)}{c_{+}(\tau)}\right)+O(\tau) \\
& \geq-\frac{1}{\tau} \int_{0}^{\tau} f^{+}\left(y_{+}(s), w(s), \alpha(s)\right) \mathrm{d} s\left(\frac{2\left\langle D \phi(\xi), \chi_{\tau}\right\rangle+2\left[D^{2} \phi(\xi)\right]\left(\chi_{\tau}, \chi_{\tau}\right)}{c_{+}(\tau)}\right)^{-}+O(\tau) \\
& =-\frac{1}{\tau} \int_{0}^{\tau} f^{+}\left(y_{+}(s), w(s), \alpha(s)\right)\left(T^{u}\left[D \phi(\xi), D^{2} \phi(\xi)\right](y, \xi)\right)^{-} \mathrm{d} s+O(\tau) .
\end{aligned}
$$

On the other hand, if (4.21) does not hold, then $\xi_{+}[\tau]=\xi$ for all $\tau$, and

$$
\begin{aligned}
\lim _{\tau \rightarrow 0^{+}} \frac{\phi\left(\xi_{\tau}\right)-\phi(\xi)}{\tau} & =0 \\
& \geq-\lim _{\tau \rightarrow 0^{+}} \frac{1}{\tau} \int_{0}^{\tau} f^{+}\left(y_{+}(s), w(s), \alpha(s)\right)\left(T^{u}\left[D \phi(\xi), D^{2} \phi(\xi)\right](y, \xi)\right)^{-} \mathrm{d} s .
\end{aligned}
$$

In both cases, we get

$$
\begin{array}{r}
\lim _{\tau \rightarrow 0^{+}} \frac{\varphi(y, t)+\phi(\xi)-\varphi(y(\tau), t-\tau)-\phi\left(\xi_{\tau}\right)}{\tau} \leq \lim _{\tau \rightarrow 0^{+}}\left(-\frac{1}{\tau} \int_{0}^{\tau}\left(\varphi_{y}(y(s), t-s) f(y(s), w(s), \alpha(s))-\varphi_{t}(y(s), t-s)\right.\right. \\
\left.\left.-f^{+}\left(y_{+}(s), w(s), \alpha(s)\right)\left(T^{u}\left[D \phi(\xi), D^{2} \phi(\xi)\right](y, \xi)\right)^{-}\right) \mathrm{d} s+O(\tau)\right)
\end{array}
$$

Since $\hat{\rho}_{2}(y, \xi)=y$, we have $T^{l}\left[D \phi(\xi), D^{2} \phi(\xi)\right](y, \xi)=0$. Hence, from (4.22), we conclude in a standard way (see Bardi-Capuzzo Dolcetta [4], p. 151).

Finally, if $\hat{\rho}_{1}(y, \xi)=y$, we change the role between $y_{+}(\cdot)$ and $y_{-}(\cdot)$. 
Lemma 4.5. Let $y \in W^{1,1}(0, T)$ be given, and let us consider the functions

$$
y_{+}(t):=\max _{s \in[0, t]} y(s), \quad y_{-}(t):=\min _{s \in[0, t]} y(s) \quad t \in[0, T] .
$$

Then, for almost every $t \in[0, T]$, we have

$$
y_{+}^{\prime}(t)=\chi_{d}\left(y(t), y_{+}(t)\right)\left(y^{\prime}(t)\right)^{+}, \quad y_{-}^{\prime}(t)=-\chi_{d}\left(y(t), y_{-}(t)\right)\left(y^{\prime}(t)\right)^{-},
$$

where $\left(x_{1}, x_{2}\right) \mapsto \chi_{d}\left(x_{1}, x_{2}\right)$ is the characteristic function of $\left\{x_{1}=x_{2}\right\}$ in $\mathbb{R}^{2}$.

Proof. We sketch the proof for $y_{+}$, which is characterized as the unique element of $W^{1,1}(0, T)$ such that

$$
\begin{cases}y_{+}^{\prime}(t)\left(y(t)-y_{+}(t)+r\right) \geq 0 & \forall r \geq 0, \text { a.e. } t \in(0, T) \\ y_{+}(t) \geq y(t) & \forall t \in[0, T] \\ y_{+}(0)=y(0) . & \end{cases}
$$

Indeed, we can regard $y_{+}(\cdot)$ as the output of a particular case of the Play operator of hysteresis with input $y(\cdot)$ (see Visintin [21], and also Bagagiolo [2]). From this we get

$$
\left|y_{+}^{\prime}(t)\right| \leq\left|y^{\prime}(t)\right| \text { a.e. } t \in(0, T) .
$$

Let $t \in(0, T)$ be such that $y^{\prime}(t)$ and $y_{+}^{\prime}(t)$ exist. If $y_{+}(t)>y(t)$, then, by continuity, (4.23) holds in $t$. Otherwise, if $y_{+}(t)=y(t)$, we should have

$$
y^{\prime}(t) \geq 0 \text {. }
$$

Hence, by (4.24), (4.25) and the following, we conclude.

$$
y_{+}^{\prime}(t)=\lim _{h \rightarrow 0} \frac{y_{+}(t+h)-y_{+}(t)}{h} \geq \lim _{h \rightarrow 0} \frac{y(t+h)-y(t)}{h}=y^{\prime}(t) .
$$

\section{UNIQUENESS}

We assume the following hypothesis

$$
\begin{aligned}
& \nu(U)>0 \text { for all open set } U \subset \mathcal{P} \text {; in particular, assuming (4.8), } \\
& \forall K \subset \mathcal{P} \text { bounded } \exists c>0 \text { such that } g \geq c \text { a.e. in } K \text {. }
\end{aligned}
$$

Theorem 5.1. Let (4.8) and (5.1) hold. Let $u$ and $v$ be bounded and uniformly continuous functions on $\mathcal{O} \times[0, T] \subset \mathbb{R} \times L^{2}(\mathcal{P}, \nu) \times[0, T]$ for every $T>0$, which respectively are viscosity subsolution and viscosity supersolution of the Cauchy problem (4.12) for every $T>0$. Then

$$
u(y, \xi, t) \leq v(y, \xi, t) \quad \forall(y, \xi, t) \in \mathcal{O} \times[0,+\infty[.
$$

As usual, from (5.2), the uniqueness of the viscosity solution of (4.12) follows. We prove Theorem 5.1 using Theorem 5.2 below, which gives the same result under the following hypothesis (in the following $\sigma_{1}$ is defined as in (2.1)).

$$
\begin{aligned}
& \exists r>0 \text { such that, setting } Q_{r}:=\left\{\left(\rho_{1}, \rho_{2}\right) \in \mathcal{P} \mid 0<\sigma_{1}<r\right\}, \\
& \nu\left(Q_{r}\right)=0, \quad \nu(U)>0 \text { for every open set } U \subset \mathcal{P} \backslash Q_{r} .
\end{aligned}
$$

The hypothesis (5.3) can be assumed for instance when the support of $\mu$ is confined on a compact set of $\mathcal{P}$ (which is open). 
Theorem 5.2. Let us suppose that the hypotheses of Theorem 5.1 are satisfied, but (5.1) is replaced by (5.3). Then the thesis of Theorem 5.1 holds.

Remark 5.3. The same proof as for Theorem 5.2 shows that, whenever $u$ and $v$ are bounded and uniformly continuous in $\mathcal{O} \times[0, T]$ for every $T>0$, and whenever they are respectively sub- and supersolution of the Hamilton-Jacobi equation in (4.12) satisfying $u(y, \xi, 0) \leq v(y, \xi, 0)$ for every $(y, \xi) \in \mathcal{O}$, then $u(y, \xi, t) \leq v(y, \xi, t)$ in $\mathcal{O} \times[0,+\infty[$.

Proof. (Th. 5.1.) For every $r>0$ we consider the strip $Q_{r}$ and define two measures $\mu_{r}$ and $\nu_{r}$ on $\mathcal{P}$ by

$$
\mathrm{d} \mu_{r}:=\chi_{\mathcal{P} \backslash Q_{r}} \mathrm{~d} \mu, \quad \mathrm{d} \nu_{r}:=\chi_{\mathcal{P} \backslash Q_{r}} \mathrm{~d} \nu .
$$

Note that $\mu_{r}$ and $\nu_{r}$ satisfy (4.8) and (5.3). Moreover, for every $r>0$ we consider the Preisach operator $\mathcal{H}_{\mu_{r}}$ corresponding to the measure $\mu_{r}$. Hence, we have a family of optimal control problems $\left\{P_{r}\right\}_{r>0}$, which are given by $f, \ell, h, A$ and $\mathcal{H}_{\mu_{r}}$. Finally, we denote by the subscript $r$ every formula, quantity, equation and set corresponding to the problem $P_{r}$ and to the measure $\nu_{r}$. However, we consider $\mathcal{O}$ as independent from $r$ (see Rems. 2.6 and 4.2).

A similar argument as in Proposition 2.8, shows that, for every $(y, \xi) \in \mathcal{O}$,

$$
S_{r}(y, \xi):=\left\{\eta \in \mathcal{S} \mid(y, \eta) \in \mathcal{O}, \eta=\xi \text { in } \mathcal{P} \backslash Q_{r}\right\}
$$

is compact for the topology of $L^{2}(\mathcal{P}, \nu)$. Hence, for every $r>0$ we define

$$
u_{r}(y, \xi, t)=\max \left\{u(y, \eta, t) \mid \eta \in \mathcal{S}_{r}(y, \xi)\right\}, \quad v_{r}(y, \xi, t)=\min \left\{v(y, \eta, t) \mid \eta \in \mathcal{S}_{r}(y, \xi)\right\} .
$$

We are now going to prove three claims. First claim: for every $T>0, u_{r}, v_{r}$ are bounded and uniformly continuous in $\mathcal{O} \times[0, T] \subset \mathbb{R} \times L^{2}\left(\mathcal{P}, \nu_{r}\right) \times[0, T]$. Let us prove the claim for $v_{r}$, the other case being analogous. For every $(y, \xi, t)$ let us take an element $(y, \tilde{\xi}, t)$ where the minimum in the definition is achieved. For every $\left(y_{1}, \xi_{1}\right),\left(y_{2}, \xi_{2}\right) \in \mathcal{O}$, we can choose a function $\xi_{1_{\xi_{2}}}$ such that $\left(y_{1}, \xi_{1_{\xi_{2}}}\right) \in \mathcal{O}, \xi_{1_{\xi_{2}}}=\xi_{1}$ in $\mathcal{P} \backslash Q_{r}$ and

$$
\left\|\xi_{\xi_{\xi_{2}}}-\xi_{2}\right\|_{L^{2}\left(Q_{r}, \nu\right)} \leq \omega_{r}\left(\left|y_{1}-y_{2}\right|+\left\|\xi_{1}-\xi_{2}\right\|_{L^{2}\left(\mathcal{P} \backslash Q_{r}, \nu_{r}\right)}\right)
$$

where $\omega_{r}$ is a modulus of continuity depending only on $r$. Indeed, by (5.1) and (5.4), it certainly holds in every compact set of $\mathcal{O}$, i.e. compact with respect to $y \in \mathbb{R}$ (in particular note that the distance of the two points where $B_{\xi_{1}}$ and $B_{\xi_{2}}$ intersect the line $\sigma_{1}=r$ is uniformly controlled by $\left.\left\|\xi_{1}-\xi_{2}\right\|_{L^{2}\left(\mathcal{P} \backslash Q_{r}, \nu_{r}\right)}\right)$, and moreover, out of a compact set, if $\left|y_{1}-y_{2}\right|$ is small the $\left\|\xi_{1}-\xi_{2}\right\|_{L^{2}\left(Q_{r}, \nu\right)}$ is also small, since $\nu$ is finite on $\mathcal{P}$. Then, we have ( $\omega$ is a modulus of continuity)

$$
\begin{aligned}
v_{r}\left(y_{1}, \xi_{1}, t_{1}\right)-v_{r}\left(y_{2}, \xi_{2}, t_{2}\right) & \leq v\left(y_{1}, \xi_{\tilde{\xi}_{2}}, t_{1}\right)-v\left(y_{2}, \tilde{\xi}_{2}, t_{2}\right) \\
& \leq \omega\left(\left|y_{1}-y_{2}\right|+\left\|\xi_{\tilde{\xi}_{2}}-\tilde{\xi}_{2}\right\|_{L^{2}(\mathcal{P}, \nu)}+\left|t_{1}-t_{2}\right|\right) \\
& =\omega\left(\left|y_{1}-y_{2}\right|+\left(\left\|\xi_{1}-\xi_{2}\right\|_{L^{2}\left(\mathcal{P} \backslash Q_{r}, \nu_{r}\right)}^{2}+\left\|\xi_{\tilde{\xi}_{2}}-\tilde{\xi}_{2}\right\|_{L^{2}\left(Q_{r}, \nu\right)}^{2}\right)^{1 / 2}+\left|t_{1}-t_{2}\right|\right),
\end{aligned}
$$

and the uniform continuity of $v_{r}$ follows. The boundedness is obvious.

Second claim: $u_{r}$ and $v_{r}$ respectively pointwise converge to $u$ and $v$ on $\mathcal{O} \times\left[0,+\infty\left[\right.\right.$ as $r \rightarrow 0^{+}$. It follows from the uniform continuity of $u, v$ and the fact that $\|\xi-\eta\|_{L^{2}(\mathcal{P}, \nu)}=O(r)$, uniformly with respect to $\eta \in S_{r}(y, \xi)$.

Third claim: $u_{r}$ and $v_{r}$ are respectively sub- and super-solution of the Hamilton-Jacobi equation of the Cauchy problem $(4.12)_{r}$. We prove that $v_{r}$ is supersolution. Let $\left.\left.(y, \xi, t) \in \mathcal{O} \times\right] 0, T\right]$ be a minimum point in 
$\left.\left.\mathcal{O} \times] 0, T] \subset \mathbb{R} \times L^{2}\left(\mathcal{P}, \nu_{r}\right) \times\right] 0, T\right]$ for $v_{r}-\varphi-\phi_{r}$ with $\left.\left.\varphi \in C^{1}(\mathbb{R} \times] 0, T\right]\right)$ and $\phi_{r} \in \mathcal{V}_{r}$. First of all note that the function

$$
\phi: L^{2}(\mathcal{P}, \nu) \rightarrow \mathbb{R}, \quad \eta \mapsto \phi_{r}\left(\chi_{\mathcal{P} \backslash Q_{r}} \eta\right)
$$

belongs to $\mathcal{V}$ and moreover for every $(y, \xi) \in \mathcal{O}$ we have

$$
\begin{aligned}
& T_{r}^{u}\left[D_{r} \phi_{r}(\xi), D_{r}^{2} \phi_{r}(\xi)\right](y, \xi)=T^{u}\left[D \phi(\xi), D^{2} \phi(\xi)\right](y, \xi), \\
& T_{r}^{l}\left[D_{r} \phi_{r}(\xi), D_{r}^{2} \phi_{r}(\xi)\right](y, \xi)=T^{l}\left[D \phi(\xi), D^{2} \phi(\xi)\right](y, \xi) .
\end{aligned}
$$

Moreover $(y, \tilde{\xi}, t)$ (see above) is a point of local minimum for $v-\varphi-\phi$ with respect to $\mathcal{O} \times] 0, T] \subset \mathbb{R} \times$ $\left.\left.L^{2}(\mathcal{P}, \nu) \times\right] 0, T\right]$. Hence, the claim is proven.

Now, for every $r>0$ we take $C(r)>0$, with $C(r) \rightarrow 0$ as $r \rightarrow 0$, such that $u_{r}(y, \xi, 0) \leq v_{r}(y, \xi, 0)+C(r)$ for every $(y, \xi) \in \mathcal{O}$ (this is possible for the definitions of $u_{r}$ and $v_{r}$, and since the density of $\nu$ is bounded). Since $v_{r}+C(r)$ is still a supersolution, by Remark 5.3 we have $u_{r} \leq v_{r}+C(r)$ in $\mathcal{O} \times[0,+\infty[$. By the claims above, we then get:

$$
u(y, \xi, t) \leq v(y, \xi, t) \forall(y, \xi, t) \in \mathcal{O} \times[0,+\infty[.
$$

Proof of Theorem 5.2. This proof is mainly concerning the treatment of the infinite dimensional part of the equation. For standard techniques in the comparison result for viscosity solutions of finite dimensional Hamilton-Jacobi equations, we refer to Bardi-Capuzzo Dolcetta [4].

By contradiction, let $T>0$ be such that

$$
m:=\sup _{\mathcal{O} \times[0, T]}(u-v)>0 .
$$

By virtue of Proposition 2.8, it is enough to consider two different cases: 1) the supremum in (5.6) is achieved on a point $(\bar{y}, \bar{\xi}, \bar{t}) \in \mathcal{O} \times[0, T] ; 2)$ there is a sequence $\left\{\left(y_{\delta}, \xi_{\delta}, t_{\delta}\right)\right\}_{\delta>0} \subseteq \mathcal{O} \times[0, T]$, with $\left|y_{\delta}\right| \rightarrow+\infty$ as $\delta \rightarrow 0^{+}$, and

$$
u\left(y_{\delta}, \xi_{\delta}, t_{\delta}\right)-v\left(y_{\delta}, \xi_{\delta}, t_{\delta}\right) \geq m-\delta>0 .
$$

Case 1). We consider two sub-cases: a) $\hat{\rho}_{2}(\bar{y}, \bar{\xi})=\bar{y}$; b) $\hat{\rho}_{1}(\bar{y}, \bar{\xi})=\bar{y}$.

Sub-case a). We use the following notations: $\|\cdot\|:=\|\cdot\|_{L^{2}(\mathcal{P}, \nu)}$, and $\{\xi=1\}:=\{\rho \in \mathcal{P} \mid \xi(\rho)=1\}$ (similarly for $\{\xi=-1\})$. We define

$$
\psi(\xi):=\mathrm{e}^{-\int_{\{\bar{\xi}=-1\}} \bar{\xi} \mathrm{d} \nu}-\mathrm{e}^{-\int_{\{\bar{\xi}=-1\}} \xi \mathrm{d} \nu}-2 \int_{\{\bar{\xi}=-1\}}(1+\xi) \mathrm{d} \nu+\int_{\mathcal{P}}(\bar{\xi}-\xi)^{2} \mathrm{~d} \nu
$$

Hence $\psi \in \mathcal{V}$. We claim that there exists a constant $c>0$ such that

$$
\psi(\xi) \geq c\|\xi-\bar{\xi}\|^{2} \quad \forall \xi \in \mathcal{S} .
$$

Indeed, the claim follows from the following relations (note that $-a \leq b \leq a \Rightarrow \mathrm{e}^{a}-\mathrm{e}^{b} \geq \mathrm{e}^{-a}(a-b)$ )

$$
\begin{aligned}
& \mathrm{e}^{-\int_{\{\bar{\xi}=-1\}} \bar{\xi} \mathrm{d} \nu}-\mathrm{e}^{-\int_{\{\bar{\xi}=-1\}} \xi \mathrm{d} \nu} \geq \frac{\mathrm{e}^{\int_{\{\bar{\xi}=-1\}} \bar{\xi} \mathrm{d} \nu}}{2} \int_{\{\bar{\xi}=-1\} \cap\{\xi=1\}}(\xi-\bar{\xi})^{2} \mathrm{~d} \nu ; \\
& -2 \int_{\{\bar{\xi}=-1\}}(1+\xi) \mathrm{d} \nu+\int_{\mathcal{P}}(\bar{\xi}-\xi)^{2} \mathrm{~d} \nu=\int_{\{\bar{\xi}=1\} \cap\{\xi=-1\}}(\xi-\bar{\xi})^{2} \mathrm{~d} \nu .
\end{aligned}
$$


For every $\varepsilon>0$, on $(\mathcal{O} \times[0, T]) \times(\mathcal{O} \times[0, T])$, we define the function

$$
\begin{aligned}
\Psi\left(y_{1}, \xi_{1}, t_{1}, y_{2}, \xi_{2}, t_{2}\right):=u\left(y_{1}, \xi_{1}, t_{1}\right)-v\left(y_{2}, \xi_{2}, t_{2}\right)-\frac{\left|y_{1}-y_{2}\right|^{2}}{\varepsilon} & \\
& -\frac{1}{\varepsilon}\left(\psi\left(\xi_{1}\right)+\psi\left(\xi_{2}\right)\right)-\frac{\left|t_{1}-t_{2}\right|^{2}}{\varepsilon}-\left|y_{1}-\bar{y}\right|^{2}-\beta\left(b\left(y_{1}\right)+b\left(y_{2}\right)\right),
\end{aligned}
$$

where $b: \mathbb{R} \rightarrow \mathbb{R}$ is nonnegative, differentiable with bounded derivative, $b(\bar{y})=0$, and $b(y) \rightarrow+\infty$ as $|y| \rightarrow+\infty$; $\beta$ is a positive parameter. We have

$$
\Psi(\bar{y}, \bar{\xi}, \bar{t}, \bar{y}, \bar{\xi}, \bar{t})=u(\bar{y}, \bar{\xi}, \bar{t})-v(\bar{y}, \bar{\xi}, \bar{t})=m>0,
$$

and, using the boundedness of $u$ and $v$, for $\left|y_{1}\right|,\left|y_{2}\right|$ large enough, we have

$$
\Psi\left(y_{1}, \xi_{1}, t_{1}, y_{2}, \xi_{2}, t_{2}\right)<0
$$

Hence (see Prop. 2.8), $\Psi$ has a positive maximum on $(\mathcal{O} \times[0, T]) \times(\mathcal{O} \times[0, T])$. Let $\left(y_{1}^{\varepsilon}, \xi_{1}^{\varepsilon}, t_{1}^{\varepsilon}, y_{2}^{\varepsilon}, \xi_{2}^{\varepsilon}, t_{2}^{\varepsilon}\right)$ be such a maximum point. By (5.8), and by standard techniques, we obtain

$$
\begin{aligned}
& \frac{\left|y_{1}^{\varepsilon}-y_{2}^{\varepsilon}\right|^{2}}{\varepsilon}+c \frac{\left\|\xi_{1}^{\varepsilon}-\bar{\xi}\right\|^{2}}{\varepsilon}+c \frac{\left\|\xi_{2}^{\varepsilon}-\bar{\xi}\right\|^{2}}{\varepsilon}+\frac{\left|t_{1}^{\varepsilon}-t_{2}^{\varepsilon}\right|^{2}}{\varepsilon}+\left|y_{1}^{\varepsilon}-\bar{y}\right|^{2} \leq \\
& \omega\left(\left|y_{1}^{\varepsilon}-y_{2}^{\varepsilon}\right|+\left\|\xi_{1}^{\varepsilon}-\xi_{2}^{\varepsilon}\right\|+\left|t_{1}^{\varepsilon}-t_{2}^{\varepsilon}\right|\right) \leq M
\end{aligned}
$$

with $\omega$ modulus of continuity. Hence, for $\varepsilon \rightarrow 0^{+}$, we have

$$
\max \left(\frac{\left|y_{1}^{\varepsilon}-y_{2}^{\varepsilon}\right|^{2}}{\varepsilon}, \frac{\left\|\xi_{1}^{\varepsilon}-\bar{\xi}\right\|^{2}}{\varepsilon}, \frac{\left\|\xi_{2}^{\varepsilon}-\bar{\xi}\right\|^{2}}{\varepsilon}, \frac{\left|t_{1}^{\varepsilon}-t_{2}^{\varepsilon}\right|^{2}}{\varepsilon},\left|y_{1}^{\varepsilon}-\bar{y}\right|^{2}\right) \rightarrow 0
$$

By (5.11), $y_{1}^{\varepsilon}$ and $y_{2}^{\varepsilon}$ tend to $\bar{y}$, and, since $\hat{\rho}_{2}(\bar{y}, \bar{\xi})=\bar{y}$, by (5.3), we can suppose (recall (5.3) and Rem. 2.7)

$$
\hat{\rho}_{2}\left(y_{i}^{\varepsilon}, \xi_{i}^{\varepsilon}\right)=y_{i}^{\varepsilon} \text { for } i=1,2 \text { and } \varepsilon \text { sufficiently small. }
$$

Indeed, if (5.12) is not possible, then $\left\|\xi_{i}^{\varepsilon}-\bar{\xi}\right\| \not \rightarrow 0$ : there is a triangle in $\mathcal{P} \backslash Q_{r}$ where $\left|\xi_{i}^{\varepsilon}-\bar{\xi}\right|=2$ for all $\varepsilon$ (the triangle $T_{2}$ in Fig. 5).

As usual, $\left(y_{1}^{\varepsilon}, \xi_{1}^{\varepsilon}, t_{1}^{\varepsilon}\right)$ is of maximum in $\left.\left.\mathcal{O} \times\right] 0, T\right]$ (note that $t_{i}^{\varepsilon}>0$ ) for

$$
(y, \xi, t) \mapsto u(y, \xi, t)-\frac{\left|y-y_{2}^{\varepsilon}\right|^{2}}{\varepsilon}-\frac{\psi(\xi)}{\varepsilon}-\frac{\left|t-t_{2}^{\varepsilon}\right|^{2}}{\varepsilon}-|y-\bar{y}|^{2}-\beta b(y),
$$

as well as $\left(y_{2}^{\varepsilon}, \xi_{2}^{\varepsilon}, t_{2}^{\varepsilon}\right)$ is a minimum point in $\left.\left.\mathcal{O} \times\right] 0, T\right]$ for

$$
(y, \xi, t) \mapsto v(y, \xi, t)+\frac{\left|y-y_{1}^{\varepsilon}\right|^{2}}{\varepsilon}+\frac{\psi(\xi)}{\varepsilon}+\frac{\left|t-t_{1}^{\varepsilon}\right|^{2}}{\varepsilon}+\beta b(y) .
$$

By (5.12), we have $T^{l}\left[D \psi\left(\xi_{i}^{\varepsilon}\right), D^{2} \psi\left(\xi_{i}^{\varepsilon}\right)\right]\left(y_{i}^{\varepsilon}, \xi_{i}^{\varepsilon}\right)=0$ for $i=1,2$ and small $\varepsilon$ (this is the main reason for which we introduced the hypothesis (5.3)). The exponential terms of $\psi$ do not give contributions to $T^{u}$. Hence, see (4.5),

$$
\begin{aligned}
T^{u}\left[D \frac{\psi}{\varepsilon}\left(\xi_{i}^{\varepsilon}\right), D^{2} \frac{\psi}{\varepsilon}\left(\xi_{i}^{\varepsilon}\right)\right]\left(y_{i}^{\varepsilon}, \xi_{i}^{\varepsilon}\right) & =\lim _{s \rightarrow 0^{+}}-\frac{4}{\varepsilon s}\left(\nu\left(P_{s}^{u}\left(y_{i}^{\varepsilon}, \xi_{i}^{\varepsilon}\right) \cap\{\bar{\xi}=-1\}\right)+\int_{P_{s}^{u}\left(y_{i}^{\varepsilon}, \xi_{i}^{\varepsilon}\right)} \bar{\xi} \mathrm{d} \nu\right) \\
& =\lim _{s \rightarrow 0^{+}}-\frac{4}{\varepsilon s} \nu\left(P_{s}^{u}\left(y_{i}^{\varepsilon}, \xi_{i}^{\varepsilon}\right) \cap\{\bar{\xi}=1\}\right) \leq 0 .
\end{aligned}
$$




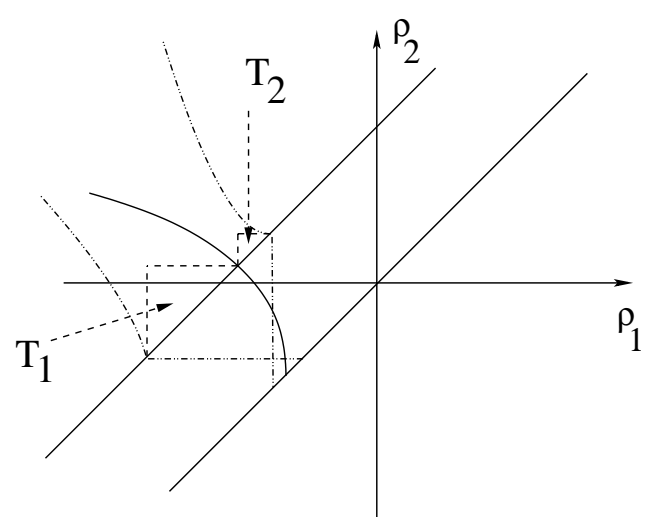

FIGURE 5. Graphic explanation for the proof of Theorem 5.2. The filled graph $\bar{\xi}$ is such that $\hat{\rho}_{1}(\bar{y}, \bar{\xi})=\hat{\rho}_{2}(\bar{y}, \bar{\xi})=\bar{y}$.

Hence, in the Hamilton-Jacobi equation, the terms involving $T^{u}$ and $T^{l}$ vanish (note that $\psi$ appears with different signs in (5.13) and (5.14)). We can then apply standard techniques to the "finite dimensional" part of the equation, and get the contradiction.

Sub-case b). In this sub-case we define the function $\gamma \in \mathcal{V}$

$$
\gamma(\xi):=\mathrm{e}^{\int_{\{\bar{\xi}=1\}} \bar{\xi} \mathrm{d} \nu}-\mathrm{e}^{\int_{\{\bar{\xi}=1\}} \xi \mathrm{d} \nu}-2 \int_{\{\bar{\xi}=1\}}(1-\xi) \mathrm{d} \nu+\int_{\mathcal{P}}(\xi-\bar{\xi})^{2} \mathrm{~d} \nu
$$

There exists $c>0$ such that (replacing $\psi$ with $\gamma$ ) (5.8) holds. We then define

$$
\begin{aligned}
\Gamma\left(y_{1}, \xi_{1}, t_{1}, y_{2} . \xi_{2}, t_{2}\right):=u\left(y_{1}, \xi_{1}, t_{1}\right)-v\left(y_{2}, \xi_{2}, t_{2}\right) & -\frac{\left|y_{1}-y_{2}\right|^{2}}{\varepsilon} \\
& -\frac{1}{\varepsilon}\left(\gamma\left(\xi_{1}\right)+\gamma\left(\xi_{2}\right)\right)-\frac{\left|t_{1}-t_{2}\right|^{2}}{\varepsilon}-\left|y_{1}-\bar{y}\right|^{2}-\beta\left(b\left(y_{1}\right)+b\left(y_{2}\right)\right) .
\end{aligned}
$$

As before, $\Gamma$ has a local maximum point $\left(y_{1}^{\varepsilon}, \xi_{1}^{\varepsilon}, t_{1}^{\varepsilon}, y_{2}^{\varepsilon}, \xi_{2}^{\varepsilon}, t_{2}^{\varepsilon}\right)$ in $\left.\left.\left.\left.(\mathcal{O} \times] 0, T\right]\right) \times(\mathcal{O} \times] 0, T\right]\right)$, with the same convergence as in (5.11). Moreover, we have $\hat{\rho}_{1}\left(y_{i}, \xi_{i}\right)=y_{i}$, and $T^{l}\left[D \gamma\left(\xi_{i}^{\varepsilon}\right), D^{2} \gamma\left(\xi_{i}^{\varepsilon}\right)\right]\left(y_{i}^{\varepsilon}, \xi_{i}^{\varepsilon}\right) \geq 0$ for $i=1,2$ and small $\varepsilon$ (see the triangle $T_{1}$ in Fig. 5). We then get the conclusion as in the sub-case a).

Case 2). For every $\varepsilon, \delta>0$, on $(\mathcal{O} \times[0, T]) \times(\mathcal{O} \times[0, T])$, we define

$$
\begin{aligned}
\Lambda\left(y_{1}, \xi_{1}, t_{1}, y_{2}, \xi_{2}, t_{2}\right):=u\left(y_{1}, \xi_{1}, t_{1}\right)-v\left(y_{2}, \xi_{2}, t_{2}\right) & -\frac{\left|y_{1}-y_{2}\right|^{2}}{\varepsilon} \\
& -\frac{\left\|\xi_{1}-\xi_{2}\right\|^{2}}{\varepsilon}-\frac{\left|t_{1}-t_{2}\right|^{2}}{\varepsilon}-\left|y_{1}-y_{\delta}\right|^{2}-\beta\left(b_{\delta}\left(y_{1}\right)+b_{\delta}\left(y_{2}\right)\right),
\end{aligned}
$$

where $b_{\delta}(y)$ is positive with derivatives uniformly bounded with respect to $\delta, b_{\delta}(y) \rightarrow+\infty$ as $|y| \rightarrow+\infty$ and $b_{\delta}\left(y_{\delta}\right)=0$. Again, $\Lambda$ has a maximum point $\left(y_{1}^{\varepsilon, \delta}, \xi_{1}^{\varepsilon, \delta}, t_{1}^{\varepsilon, \delta}, y_{1}^{\varepsilon, \delta}, \xi_{1}^{\varepsilon, \delta}, t_{1}^{\varepsilon, \delta}\right)$ on $\left.\left.\left.\left.(\mathcal{O} \times] 0, T\right]\right) \times(\mathcal{O} \times] 0, T\right]\right)$, and as $\max \{\varepsilon, \delta\} \rightarrow 0$

$$
\max \left(\frac{\left|y_{1}^{\varepsilon, \delta}-y_{2}^{\varepsilon, \delta}\right|^{2}}{\varepsilon}, \frac{\left\|\xi_{1}^{\varepsilon, \delta}-\xi_{2}^{\varepsilon, \delta}\right\|^{2}}{\varepsilon}, \frac{\left|t_{1}^{\varepsilon}-t_{2}^{\varepsilon}\right|^{2}}{\varepsilon},\left|y_{1}^{\varepsilon, \delta}-y_{\delta}\right|^{2}\right) \rightarrow 0 .
$$

Since $\left|y_{\delta}\right| \rightarrow+\infty$ as $\delta \rightarrow 0$, and $\nu(\mathcal{P})<+\infty$, for every fixed $\varepsilon>0$ we can find $\delta_{\varepsilon}>0$ such that for $\delta \leq \delta_{\varepsilon}$ we have $\nu\left(\left\{\rho \in \mathcal{P}\left|\rho_{2} \geq\right| y_{\delta} \mid\right\}\right) \leq \varepsilon^{2}$ and $\nu\left(\left\{\rho \in \mathcal{P}\left|\rho_{1} \leq-\right| y \delta \mid\right\}\right) \leq \varepsilon^{2}$. Moreover we can suppose that $\delta_{\varepsilon} \rightarrow 0$ as 
$\varepsilon \rightarrow 0$. We then recover two test functions $\zeta_{1}$ and $\zeta_{2}$ (depending on $\varepsilon$ ) such that $\left(y_{i}^{\varepsilon, \delta}, \xi_{i}^{\varepsilon, \delta}, t_{i}^{\varepsilon, \delta}\right)$ is, respectively for $i=1,2$, a point of local maximum and local minimum for $u-\zeta_{1}$ and $v-\zeta_{2}$, and

$$
\delta \leq \delta_{\varepsilon} \Rightarrow\left|T^{j}\left[D \zeta_{i}\left(\xi_{i}^{\varepsilon, \delta}\right), D^{2} \zeta_{i}\left(\xi_{i}^{\varepsilon, \delta}\right)\right]\left(y_{i}^{\varepsilon, \delta}, \xi_{i}^{\varepsilon, \delta}\right)\right| \leq \varepsilon . \forall i=1,2 \forall j=u, l .
$$

Hence, the "infinite dimensional" terms are infinitesimal as $\varepsilon \rightarrow 0$. We then obtain the desired contradiction applying standard techniques to the remaining "finite dimensional" part of the equation.

Remark 5.4. The hypotheses (5.1) and (5.3) are probably not necessary for the uniqueness result. Moreover, the hypothesis (5.3) can be weakened, considering not a strip $Q_{r}$ but other geometrical situation. However, we point out again that $\nu$ is not a datum of the control problem, but, after (2.5), its choice is at our disposal, in order to fit the problem in a suitable functional space.

\section{The vectorial CASE}

In this section we give a possible extension of the control problem. In particular, such extension covers the case of a controlled hysteresis dynamical system in $\mathbb{R}^{n}$, where the hysteresis is given by a weighted average of a finite number of Preisach operators, each one of them having, as input, a suitable function of the state $y \in \mathbb{R}^{n}$. The simplest example is given by one Preisach operator only, which acts on the evolution of a fixed component of the input $y \in \mathbb{R}^{n}$. This is indeed the situation in $[6-8,15,19]$, possibly with a hysteresis model different from the Preisach one. However, we set the problem in a very general way.

When non specifically stated, the notations are the same as in the previous sections. Let $n, m$, and $r$ be nonnegative integers. Let us consider the functions

$$
\begin{aligned}
& f: \mathbb{R}^{n} \times \mathbb{R}^{m} \times A \rightarrow \mathbb{R}^{n}, \quad G: \mathbb{R}^{r} \rightarrow \mathbb{R}^{m}, \quad g_{i}: \mathbb{R}^{n} \rightarrow \mathbb{R}, i=1, \ldots, r, \\
& \ell: \mathbb{R}^{n} \times \mathbb{R}^{m} \times A \rightarrow\left[0,+\infty\left[, \quad h: \mathbb{R}^{n} \times \mathbb{R}^{m} \rightarrow[0,+\infty[.\right.\right.
\end{aligned}
$$

The hypotheses are: $f$ is bounded, continuous, and Lipschitz continuous with respect to its first two entries, uniformly with respect to the third one; $G$ is Lipschitz continuous; for every $i, g_{i}$ is $C^{1} ; \ell$ is bounded, continuous, and uniformly continuous with respect to its first two entries, uniformly with respect to the third one; $h$ is bounded and uniformly continuous. Moreover, for every $i=1, \ldots, r$, we have a pair of measures $\left(\mu_{i}, \nu_{i}\right)$ on the Preisach plane, which, pair by pair, satisfy all the hypotheses as the pair $(\mu, \nu)$ in the previous sections. Finally we define

$$
\tilde{\mathcal{O}}:=\left\{(y, \xi)=\left(y, \xi_{1}, \ldots, \xi_{r}\right) \in \mathbb{R}^{n} \times \mathcal{S} \times \cdots \times \mathcal{S} \mid\left(g_{i}(y), \xi_{i}\right) \in \mathcal{O} \forall i=1, \ldots, r\right\},
$$

and we equipped it with the graph-norm of $\mathbb{R}^{n} \times L^{2}\left(\mathcal{P}, \nu_{1}\right) \times \cdots \times L^{2}\left(\mathcal{P}, \nu_{r}\right)$.

We consider the optimal control problem given by

$$
\begin{cases}y^{\prime}(t)=f(y(t), w(t), \alpha(t)) & t>0 \\ w(t)=G\left(w_{1}(t), \ldots, w_{r}(t)\right) & t \geq 0 \\ w_{i}(t)=\mathcal{H}_{\mu_{i}}\left[g_{i} \circ y, \xi_{i 0}\right](t) & t \geq 0, i=1, \ldots, r \\ y(0)=y_{0} & \\ \left(y_{0}, \xi_{0}\right)=\left(y_{0}, \xi_{10}, \ldots, \xi_{r 0}\right) \in \tilde{\mathcal{O}} & \end{cases}
$$

The cost functional and the value function are, respectively

$$
\begin{aligned}
J\left(y_{0}, \xi_{0}, t, \alpha\right) & :=\int_{0}^{t} \mathrm{e}^{-\lambda s} \ell(y(s), w(s), \alpha(s)) \mathrm{d} s+\mathrm{e}^{-\lambda t} h(y(t), w(t)), \\
V\left(y_{0}, \xi_{0}, t\right) & =\inf _{\alpha \in \mathcal{A}} J\left(y_{0}, \xi_{0}, t, \alpha\right) .
\end{aligned}
$$


Let $\mathcal{L}_{i}$ be the space of symmetric bounded bilinear forms on $L^{2}\left(\mathcal{P}, \nu_{i}\right)$. For every $(y, \xi) \in \tilde{\mathcal{O}}$, and every collection of pairs $\left(\psi_{i}, \pi_{i}\right)_{i=1, \ldots, r},\left(\psi_{i}, \pi_{i}\right) \in L^{2}\left(\mathcal{P}, \nu_{i}\right) \times \mathcal{L}_{i}$, we consider the collections of upper and left limits $\left(T_{i}^{u}\left[\psi_{i}, \pi_{i}\right]\left(g_{i}(y), \xi_{i}\right)\right)_{i}$ and $\left(T_{i}^{l}\left[\psi_{i}, \pi_{i}\right]\left(g_{i}(y), \xi_{i}\right)\right)_{i}$ where, for every $i=1, \ldots, r$, the limits are calculated as in the previous sections, but with respect to the topology of $L^{2}\left(\mathcal{P}, \nu_{i}\right)$, respectively.

We consider the following infinite dimensional hamiltonians, defined on (a subset of)

$$
\tilde{\mathcal{O}} \times \mathbb{R}^{n} \times\left(L^{2}\left(\mathcal{P}, \nu_{1}\right) \times \mathcal{L}_{1}\right) \times \cdots \times\left(L^{2}\left(\mathcal{P}, \nu_{r}\right) \times \mathcal{L}_{r}\right),
$$

by (here $w=G\left(w_{1}, \ldots, w_{r}\right)$, where $w_{i}=\int_{\mathcal{P}} \xi_{i} \mathrm{~d} \mu_{i}$; and $\nabla$ is the gradient with respect to $y \in \mathbb{R}^{n}$ )

$$
\begin{aligned}
H\left(y, \xi, p,\left(\psi_{1}, \pi_{1}\right), \ldots,\left(\psi_{r}, \pi_{r}\right)\right):= & \sup _{a \in A}\left\{-p \cdot f(y, w, a)-\sum_{i=1}^{r}\left[\left(\nabla g_{i}(y) \cdot f(y, w, a)\right)^{+} T_{i}^{u}\left[\psi_{i}, \pi_{i}\right]\left(g_{i}(y), \xi_{i}\right)\right.\right. \\
& \left.\left.-\left(\nabla g_{i}(y) \cdot f(y, w, a)\right)^{-} T_{i}^{l}\left[\psi_{i}, \pi_{i}\right]\left(g_{i}(y), \xi_{i}\right)\right]-\ell(y, w, a)\right\} \\
H^{-}\left(y, \xi, p,\left(\psi_{1}, \pi_{1}\right), \ldots,\left(\psi_{r}, \pi_{r}\right)\right):= & \sup _{a \in A}\left\{-p \cdot f(y, w, a)-\sum_{i=1}^{r}\left[\left(\nabla g_{i}(y) \cdot f(y, w, a)\right)^{+}\left(T_{i}^{u}\left[\psi_{i}, \pi_{i}\right]\left(g_{i}(y), \xi_{i}\right)\right)^{+}\right.\right. \\
& \left.\left.+\left(\nabla g_{i}(y) \cdot f(y, w, a)\right)^{-}\left(T_{i}^{l}\left[\psi_{i}, \pi_{i}\right]\left(g_{i}(y), \xi_{i}\right)\right)^{-}\right]-\ell(y, w, a)\right\} \\
H^{+}\left(y, \xi, p,\left(\psi_{1}, \pi_{1}\right), \ldots,\left(\psi_{r}, \pi_{r}\right)\right):= & \sup _{a \in A}\left\{-p \cdot f(y, w, a)+\sum_{i=1}^{r}\left[\left(\nabla g_{i}(y) \cdot f(y, w, a)\right)^{+}\left(T_{i}^{u}\left[\psi_{i}, \pi_{i}\right]\left(g_{i}(y), \xi_{i}\right)\right)^{-}\right.\right. \\
& \left.\left.+\left(\nabla g_{i}(y) \cdot f(y, w, a)\right)^{-}\left(T_{i}^{l}\left[\psi_{i}, \pi_{i}\right]\left(g_{i}(y), \xi_{i}\right)\right)^{+}\right]-\ell(y, w, a)\right\} .
\end{aligned}
$$

Theorem 6.1. For every $T>0$, the value function is the unique bounded and uniformly continuous viscosity solution of the following Cauchy problem

$$
\left\{\begin{array}{l}
\left.\left.V_{t}(y, \xi, t)+\lambda V(y, \xi, t)+H\left(y, \xi, \nabla V(y, \xi, t), D V(y, \xi, t), D^{2} V(y, \xi, t)\right)=0 \quad \text { in } \tilde{\mathcal{O}} \times\right] 0, T\right] \\
V(y, \xi, 0)=h(y, w) .
\end{array}\right.
$$

The definition of viscosity solution is the following. For every $i$, let $\mathcal{V}_{i}$ be the set of test functions as in (4.3), where we have replaced $\nu, T^{u}$, and $T^{l}$ by $\nu_{i}, T_{i}^{u}$, and $T_{i}^{l}$ respectively. Then, for every choice of the test functions $\varphi \in C^{1}\left(\mathbb{R}^{n} \times\right] 0,+\infty[), \phi_{i} \in \mathcal{V}_{i}, i=1, \ldots, r$, we have the following:

$1)$ if $V(y, \xi, t)-\varphi(y, t)-\phi_{1}\left(\xi_{1}\right)-\cdots-\phi_{r}\left(\xi_{r}\right)$ has a local maximum at $(y, \xi, t)$ in $\left.\tilde{\mathcal{O}} \times\right] 0,+\infty[$, then

$$
\varphi_{t}(y, t)+\lambda V(y, \xi, t)+H^{-}\left(y, \xi, \nabla \varphi(y, t),\left(D \phi_{1}\left(\xi_{1}\right), D^{2} \phi_{1}\left(\xi_{1}\right)\right), \ldots,\left(D \phi_{r}\left(\xi_{r}\right), D^{2} \phi_{r}\left(\xi_{r}\right)\right)\right) \leq 0
$$

$2)$ if $V(y, \xi, t)-\varphi(y, t)-\phi_{1}\left(\xi_{1}\right)-\cdots-\phi_{r}\left(\xi_{r}\right)$ has a local minimum at $(y, \xi, t)$ in $\left.\tilde{\mathcal{O}} \times\right] 0,+\infty[$, then

$$
\varphi_{t}(y, t)+\lambda V(y, \xi, t)+H^{+}\left(y, \xi, \nabla \varphi(y, t),\left(D \phi_{1}\left(\xi_{1}\right), D^{2} \phi_{1}\left(\xi_{1}\right)\right), \ldots,\left(D \phi_{r}\left(\xi_{r}\right), D^{2} \phi_{r}\left(\xi_{r}\right)\right)\right) \geq 0 .
$$

Proof. (Sketched proof of Th. 6.1.) We confine this sketched proof only to the proof that $V$ is viscosity solution of the Cauchy problem. With respect to what previously done, the difference here is that we have to work with the sign of $\nabla g_{i}(y) \cdot f(y, w, a)$ and not with the sign of $f(y, w, a)$. Moreover, the function $t \mapsto g_{i}(y(t))$ is not solution of the ODE. 
Let us prove that $V$ is subsolution. By DPP (which still holds), we obtain a similar inequality as (4.15), where we now have the $r$ differences $\phi_{i}\left(\xi_{i}\right)-\phi_{i}\left(\xi_{i \tau}\right)$. Again, the problem is passing to the limit as $\tau \rightarrow 0^{+}$in $\left(\phi_{i}\left(\xi_{i}\right)-\phi_{i}\left(\xi_{i} \tau\right)\right) / \tau$. Note that the evolution of $\xi_{i}$ is governed by the evolution of $g_{i} \circ y$. Again, we have some cases.

a) $\nabla g_{i}(y) \cdot f(y, w, a)=0$. This does not imply that $g_{i}(y(t)) \equiv g_{i}(y)$ (and hence $\xi_{i \tau} \equiv \xi_{i}$ ), since we are not working with the solution of an ODE. However, we have $g_{i}(y(\tau))-g_{i}(y)=o(\tau)$, and hence $\left\|\xi_{i \tau}-\xi_{i}\right\|_{L^{2}\left(\mathcal{P}, \nu_{i}\right)}^{2}=$ $2\left\|\xi_{i \tau}-\xi_{i}\right\|_{L^{1}\left(\mathcal{P}, \nu_{i}\right)}=o(\tau)$. Hence, using the second order Taylor's formula for $\phi_{i}$ and recalling that, by hypothesis, $D \phi_{i}\left(\xi_{i}\right) \in L^{\infty}\left(\mathcal{P}, \nu_{i}\right)$, we get

$$
\begin{aligned}
\lim _{\tau \rightarrow 0^{+}} \frac{\phi_{i}\left(\xi_{i \tau}\right)-\phi_{i}\left(\xi_{i}\right)}{\tau}= & \lim _{\tau \rightarrow 0^{+}} \frac{1}{\tau}\left(\left\langle D \phi_{i}\left(\xi_{i}\right), \xi_{i \tau}-\xi_{i}\right\rangle+\frac{1}{2}\left[D^{2} \phi_{i}\left(\xi_{i}\right)\right]\left(\xi_{i \tau}-\xi_{i}, \xi_{i \tau}-\xi_{i}\right)\right)=0 \\
= & \left(\nabla g_{i}(y) \cdot f(y, w, a)\right)^{+} T_{i}^{u}\left[D \phi_{i}\left(\xi_{i}\right), D^{2} \phi_{i}\left(\xi_{i}\right)\right]\left(g_{i}(y), \xi_{i}\right) \\
& -\left(\nabla g_{i}(y) \cdot f(y, w, a)\right)^{-} T_{i}^{l}\left[D \phi_{i}\left(\xi_{i}\right), D^{2} \phi_{i}\left(\xi_{i}\right)\right]\left(g_{i}(y), \xi_{i}\right) .
\end{aligned}
$$

b) $\nabla g_{i}(y) \cdot f(y, w, a)>0\left(\right.$ resp. $\left.\nabla g_{i}(y) \cdot f(y, w, a)<0\right)$. Then, at least for small times, the quantity $g_{i}(y(\tau))$ is increasing (resp. decreasing). Hence, we can argue as before, replacing (4.17) (resp. (4.18)) by

$$
\xi_{i \tau}-\xi_{i}=2 \chi_{\mathcal{P}_{\nabla g_{i}(y) \cdot f(y, w, a) \tau}^{u}}\left(g_{i}(y), \xi_{i}\right)+\sigma(\tau), \quad\left(\text { resp. by } \xi_{i \tau}-\xi_{i}=-2 \chi_{\mathcal{P}_{-\nabla g_{i}(y) \cdot f(y, w, a) \tau}^{l}\left(g_{i}(y), \xi_{i}\right)}+\sigma(\tau)\right),
$$

and we obtain the conclusion.

To prove that $V$ is a supersolution, using Lemma 4.5 and its notations, we can replace (4.20) by $\xi_{i \tau}=$ $\xi_{i+}[\tau]+\sigma(\tau)$, where $\tau \mapsto \xi_{i+}[\tau]$ is the evolution of the internal variables of $\mathcal{H}_{\mu_{i}}\left[\left(g_{i} \circ y\right)_{+}, \xi_{i}\right](\cdot)$; and (4.21) by

$$
\left(g_{i}(y(\tau))_{+}-g_{i}(y)=\int_{0}^{\tau} \chi_{d}\left(( g _ { i } ( y ( s ) ) _ { + } , g _ { i } ( y ( s ) ) ) \left(\nabla g_{i}(y(s)) \cdot f(y(s), w(s), \alpha(s))^{+} \mathrm{d} s .\right.\right.\right.
$$

Hence, we obtain the same inequality as in (4.22), with the replacing of $f^{+}\left(y_{+}(s), w(s), \alpha(s)\right)$ by $\left(\nabla g_{i}(y(s))\right.$. $f(y(s), w(s), \alpha(s))^{+}$as major change. We then can conclude.

To prove the uniqueness result, we just repeat the proofs of Theorems 5.1 and 5.2. In particular, repeating the proof of Theorem 5.2, we have to suppose that a condition as (5.3) holds for every measure $\nu_{i}$. Moreover, we have to use the test functions $\psi_{i}$ or the test functions $\gamma_{i}, i=1 \ldots, r$ as in that proof, by separately analyzing case by case all the $r$ "components" of $\tilde{\mathcal{O}}$, i.e. $\left(g_{i}(y), \xi_{i}\right), i=1, \ldots r$.

Remark 6.2. As we already said, if the function $G$ is scalar-valued, it may represent a weighted average of a finite number of Preisach operators, with, respectively, a suitable function of the vector $y$ (for instance a component) as input. We believe that similar results to the ones presented in this work, may be obtained by a similar analysis, in the case that the finite average is replaced by a series or even by a superposition of a continuum of Preisach operators. For instance, a similar analysis as in Bagagiolo [2] may be performed. Indeed in [2], the case of the Prandtl-Ishlinskii model is studied. That model is given by the superposition of a continuum of Plays operators, which are continuous hysteresis operators (by the way, note that the Preisach model is a superposition of a continuum of delayed relays, which however are discontinuous hysteresis operators). Hence, starting from the results of the present paper, the case of a continuum of Preisach models, which are continuous hysteresis operators, may be not very complicated. However, we did not check the details. 


\section{REFERENCES}

[1] F. Bagagiolo, An infinite horizon optimal control problem for some switching systems. Discrete Contin. Dyn. Syst. Ser. B 1 (2001) 443-462.

[2] F. Bagagiolo, Dynamic programming for some optimal control problems with hysteresis. NoDEA Nonlinear Differ. Equ. Appl. 9 (2002) 149-174.

[3] F. Bagagiolo, Optimal control of finite horizon type for a multidimensional delayed switching system. Department of Mathematics, University of Trento, Preprint No. 647 (2003).

[4] M. Bardi and I. Capuzzo Dolcetta, Optimal Control and Viscosity Solutions of Hamilton-Jacobi-Bellman Equations. Birkhäuser, Boston (1997).

[5] G. Barles and P.L. Lions, Fully nonlinear Neumann type boundary conditions for first-order Hamilton-Jacobi equations. Nonlinear Anal. 16 (1991) 143-153.

[6] S.A. Belbas and I.D. Mayergoyz, Optimal control of dynamic systems with hysteresis. Int. J. Control 73 (2000) $22-28$.

[7] S.A. Belbas and I.D. Mayergoyz, Dynamic programming for systems with hysteresis. Physica B Condensed Matter 306 (2001) 200-205.

[8] M. Brokate, ODE control problems including the Preisach hysteresis operator: Necessary optimality conditions, in Dynamic Economic Models and Optimal Control, G. Feichtinger Ed., North-Holland, Amsterdam (1992) 51-68.

[9] M. Brokate and J. Sprekels, Hysteresis and Phase Transitions. Springer, Berlin (1997).

[10] M.G. Crandall and P.L. Lions, Hamilton-Jacobi equations in infinite dimensions. Part I: Uniqueness of solutions. J. Funct. Anal. 62 (1985) 379-396.

[11] E. Della Torre, Magnetic Hysteresis. IEEE Press, New York (1999).

[12] M.A. Krasnoselskii and A.V. Pokrovskii, Systems with Hysteresis. Springer, Berlin (1989). Russian Ed. Nauka, Moscow (1983).

[13] P. Krejci, Convexity, Hysteresis and Dissipation in Hyperbolic Equations. Gakkotosho, Tokyo (1996).

[14] I. Ishii, A boundary value problem of the Dirichlet type for Hamilton-Jacobi equations. Ann. Scuola Norm. Sup. Pisa Cl. Sci. 16 (1989) 105-135.

[15] S.M. Lenhart, T. Seidman and J. Yong, Optimal control of a bioreactor with modal switching. Math. Models Methods Appl. Sci. 11 (2001) 933-949.

[16] P.L. Lions, Neumann type boundary condition for Hamilton-Jacobi equations. Duke Math. J. 52 (1985) 793-820.

[17] P.L. Lions, Viscosity solutions of fully nonlinear second-order equations and optimal stochastic control in infinite dimensions. Part I: the case of bounded stochastic evolutions. Acta Math. 161 (1988) 243-278.

[18] I.D. Mayergoyz, Mathematical Models of Hysteresis. Springer, New York (1991).

[19] X. Tan and J.S. Baras, Optimal control of hysteresis in smart actuators: a viscosity solutions approach. Center for Dynamics and Control of Smart Actuators, preprint (2002).

[20] G. Tao and P.V. Kokotovic, Adaptive Control of Systems with Actuator and Sensor Nonlinearities. John Wiley \& Sons, New York (1996).

[21] A. Visintin, Differential Models of Hysteresis. Springer, Heidelberg (1994). 